\title{
The efficacy and mechanism of apoptosis induction by hypericin-mediated sonodynamic therapy in THP-I macrophages
}

This article was published in the following Dove Press journal:

International Journal of Nanomedicine

22 January 2015

Number of times this article has been viewed

\author{
Xuesong $\mathrm{Li}^{1}$,* \\ Lei $\mathrm{Gao}^{2, *}$ \\ Longbin Zheng' \\ Jiayuan Kou' \\ Xing Zhu' \\ Yueqing Jiang' \\ Zhaoyu Zhong' \\ Juhua Dan' \\ Haobo $X^{3}$ \\ Yang Yang ${ }^{3}$ \\ Hong $\mathrm{Li}^{\prime}$ \\ Sa Shi' \\ Wenwu Cao ${ }^{4,5}$ \\ Yajun Zhao' \\ Ye Tian ${ }^{1,3}$ \\ Liming Yang'
}

'Department of Pathophysiology, Harbin Medical University, Harbin, People's Republic of China; ${ }^{2}$ Electron Microscopy Centre, Harbin Medical University, Harbin, People's Republic of China; ${ }^{3}$ Division of Cardiology, The First Affiliated Hospital, Harbin Medical University, Harbin, People's Republic of China; ${ }^{4}$ Laboratory of Sono- and Photo-theranostic Technologies, Harbin Institute of Technology, Harbin, People's Republic of China; ${ }^{5}$ Materials Research Institute, The Pennsylvania State University, University Park, PA, USA

*These authors contributed equally to this work

Correspondence: Liming Yang; Ye Tian Department of Pathophysiology, Harbin Medical University, Harbin I5008I,

People's Republic of China

Tel +8645 I 86674548

Fax +8645 I 86674548

Email limingyang@ems.hrbmu.edu.cn; yetian@ems.hrbmu.edu.cn
Purpose: To investigate the sonoactivity of hypericin (HY), together with its sonodynamic effect on THP-1 macrophages and the underlying mechanism.

Materials and methods: CCK- 8 was used to examine cell viability. Confocal laser scanning microscopy was performed to assess the localization of HY in cells, reactive oxygen species (ROS) generation, and opening of the mitochondrial permeability transition pore (mPTP) after different treatments. Apoptosis was analyzed using Hoechst-propidium iodide and transmission electron microscopy. Mitochondrial membrane potential $(\Delta \Psi \mathrm{m})$ collapse was detected via fluorescence microscopy. Lipoprotein oxidation was determined in malondialdehyde (MDA) assays. Western blotting was conducted to determine the translocation of BAX and cytochrome $\mathrm{C}$ and the expression of apoptosis-related proteins.

Results: HY was sublocalized among the nuclei and the mitochondria, endoplasmic reticulum, Golgi apparatus, and lysosome in the cytosol of THP-1 macrophages. Under low-intensity ultrasound irradiation, HY significantly decreased cell viability and induced apoptosis. Furthermore, greater ROS generation, higher MDA levels, and greater $\Delta \Psi \mathrm{m}$ loss were observed in the sonodynamic therapy (SDT) group. Both ROS generation and MDA levels were significantly reduced by the ROS scavenger N-acetyl cysteine (NAC) and the singlet oxygen scavenger sodium azide. Most of the loss of $\Delta \Psi \mathrm{m}$ was inhibited by pretreatment with NAC, sodium azide, and the mPTP inhibitor cyclosporin A (CsA). mPTP opening was induced upon SDT but was reduced by pretreatment with bongkrekic acid, 4,4'-diisothiocyanatostilbene-2,2'-disulfonic acid disodium, CsA, and NAC. Western blot analyses revealed translocation of BAX and cytochrome $\mathrm{C}$, downregulated expression of $\mathrm{Bcl}-2$, and upregulated expression of cleaved caspase-9, cleaved caspase-3, and cleaved poly(ADP-ribose) polymerase in the SDT group, which were reversed by NAC.

Conclusion: HY mediated SDT-induced apoptosis in THP-1 macrophages via ROS generation. Then, the proapoptotic factor BAX translocated from the cytosol to the mitochondria, increasing the ratio of $\mathrm{BAX} / \mathrm{Bcl}-2$, and the $\mathrm{mPTP}$ opened to release cytochrome $\mathrm{C}$. This study demonstrated the great potential of HY-mediated SDT for treating atherosclerosis.

Keywords: apoptosis, hypericin, sonodynamic therapy, mitochondria-caspase pathway, atherosclerosis

\section{Introduction}

Acute cardiovascular events, the principal cause of death worldwide, pose a severe threat to human health, and are primarily caused by the rupture of atherosclerotic plaques during atherosclerosis (AS). ${ }^{1}$ Macrophages play a pivotal role in atherogenesis, and the phenotype and quantity of these cells in atherosclerotic lesions influence both disease progression and plaque regression. ${ }^{2}$ Photodynamic therapy (PDT) has been 
used to treat cardiovascular pathologies, including AS and restenosis, ${ }^{3-5}$ which produces reactive oxygen species (ROS) and photo-induced biological damage according to the principle of energy transfer from light to a photosensitizer in tissues. ${ }^{6}$ However, because of its poor tissue penetration, PDT is limited to superficial lesions. Fortunately, an alternative therapy exists: low-intensity ultrasound can penetrate deeply into tissues due to its much lower attenuation coefficient in tissues compared with lasers. ${ }^{7-11}$ To resolve the problem of penetration depth, we made use of low-intensity ultrasound as a substitute for light in this study.

Sonodynamic therapy (SDT) is an active, noninvasive approach that has been used successfully to treat various diseases and was developed on the basis of PDT, utilizing the combined effects of ultrasound and sonosensitizers. ${ }^{12-16}$ Nonthermal ultrasound reaches nonsuperficial objects to focus energy on targeted tissues, inducing local cytotoxicity via activating preloaded sonosensitizers, while minimizing undesirable injury to surrounding normal tissues.

The most critical problem in the development of SDT is the proper choice of a sonosensitizer, because the therapeutic efficacy of SDT primarily depends on the physical and chemical properties of the sonosensitizer. ${ }^{17-19}$ Most sonosensitizers originate from porphyrin-derivative photosensitizers, such as hematoporphyrin (HP), which also causes tumor cell death when irradiated by ultrasound. However, this class of sonosensitizers has several disadvantages, including high costs and high dosage requirements when used for SDT. ${ }^{20}$ Therefore, it is essential to develop a more readily available sonosensitizer that can overcome the aforementioned disadvantages.

Recently, Chinese herbs have become popular sources of sonosensitizers for SDT. Due to the phagocytic function, the sonosensitizers could be absorbed in macrophages specifically. Our group demonstrated that emodin, curcumin, and 5-aminolevolinic acid (ALA) have sonodynamic effects in macrophages when combined with ultrasound by inducing apoptosis in vitro. ${ }^{1,19,21}$ The polycyclic dione hypericin (HY), which is one of the active ingredients of St John's wort, is a photosensitizer that accumulates in dark glands found in flowers and leaves. ${ }^{22-24} \mathrm{HY}$ has been proposed as a highly promising photosensitizer due to its minimal toxicity, tumor high selectivity, high clearance from the host body, much singlet oxygen production and anti-inflammatory properties, as HY is able to induce the apoptosis of human lens epithelial cells, nasopharyngeal carcinoma cells, and U87 glioma cells $\mathrm{s}^{20,23,25-28}$ during the rapid healing process after PDT. However, the effectiveness of HY as a potential sonosensitizer for SDT remains relatively unknown.

In this study, we aimed to investigate the ability of the application of low HY concentrations with low-intensity ultrasound (HY-SDT) to induce apoptosis in THP-1 macrophages and to examine the corresponding mechanism through the mitochondria-caspase pathway via ROS production, mitochondrial membrane lipid peroxidation, mitochondrial permeability transition pore (mPTP) opening, and mitochondrial dysfunction.

\section{Materials and methods \\ Ultrasonic exposure system}

The ultrasonic generator and power amplifier (Figure 1) were developed by the Condensed Matter Science and Technology Institute of the Harbin Institute of Technology (Harbin, People's Republic of China). The transducer (diameter $3.5 \mathrm{~cm}$, resonance frequency $1.0 \mathrm{MHz}$, duty factor $10 \%$, repetition frequency $100 \mathrm{~Hz}$ ) was placed under a degassed water bath, and the cells were placed at a $12 \mathrm{~cm}$ vertical distance from the transducer. The ultrasonic intensity was $0.5 \mathrm{~W} / \mathrm{cm}^{2}$ and was measured using a hydrophone (Onda Corporation, Sunnyvale, CA, USA). During the sonication procedure, the temperature of the solution inside the cultures increased by less than $0.5^{\circ} \mathrm{C}$.

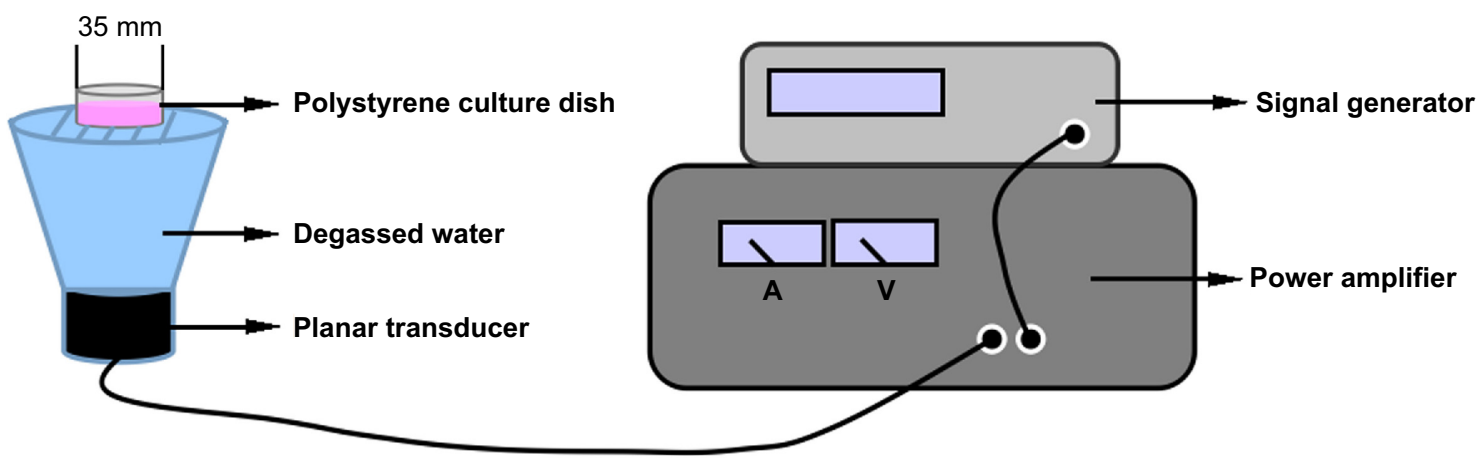

Figure I Ultrasonic exposure system.

Note: The culture dish was placed above the center of the transducer ( $1.0 \mathrm{MHz}$ center frequency, $10 \%$ duty factor), which was placed I $2 \mathrm{~cm}$ above a bath of degassed water for in vitro experiments. 


\section{Cell culture}

Human THP-1 monocytes were obtained from the American Type Culture Collection (ATCC, Manassas, VA, USA). The cells were cultured using a split ratio of 1:10 in RPMI 1640 medium (HyClone, Logan, UT, USA) supplemented with $10 \%$ fetal bovine serum (FBS, HyClone), $20 \mu \mathrm{g} / \mathrm{mL}$ penicillin, and $20 \mu \mathrm{g} / \mathrm{mL}$ streptomycin (Sigma-Aldrich Co., St Louis, MO, USA). Culture was carried out at $37^{\circ} \mathrm{C}$ in a humidified incubator containing $5 \% \mathrm{CO}_{2}$, and the medium was refreshed every 2-3 days. For the experiment, the cells were seeded at a density of $1.0 \times 10^{5}$ cells per milliliter and were differentiated into macrophages by adding $100 \mathrm{ng} / \mathrm{mL}$ phorbol-12-myristate-13-acetate (La Jolla, CA, USA) for 72 hours.

\section{SDT treatment protocols}

HY was obtained from Sigma-Aldrich Co. A $1 \mathrm{mg} / \mathrm{mL}$ stock solution in dimethyl sulfoxide was stored as aliquots at $-20^{\circ} \mathrm{C}$ in the dark. Working solutions were prepared fresh at different concentrations in 1640 medium containing 10\% FBS.

THP-1 macrophages in exponential phase were collected, differentiated, and randomly divided into four groups: 1) control, 2) ultrasound alone, 3) HY alone, and 4) SDT. For the HY and SDT groups, the cells were incubated with $0.25 \mu \mathrm{g} / \mathrm{mL}$ HY for a drug loading time of 4 hours in FBSloaded RPMI 1640 medium. Instead of HY, an equivalent volume of medium was used in the control and ultrasound alone groups. The cells in the ultrasound and SDT groups were exposed to ultrasound at a frequency and intensity of $1.0 \mathrm{MHz}$ and $0.5 \mathrm{~W} / \mathrm{cm}^{2}$, respectively. After the treatments, the cells were carefully washed once in phosphate-buffered saline (PBS), cultured in fresh medium for another 6 hours and then subjected to different analyses.

For inhibitory experiments, z-VAD-FMK (z-VAD), cyclosporin A (CsA), N-acetyl-cysteine (NAC), sodium azide $\left(\mathrm{NaN}_{3}\right)$, mannitol, superoxide dismutase (SOD), catalase (CAT), bongkrekic acid (BA), and 4,4'-diisothiocyanatostilbene-2,2'-disulfonic acid disodium (DIDS) were added to the culture medium together with HY loading for 4 hours.

\section{Cell viability}

Cell viability was assessed in a CCK-8 assay (Beyotime Biotechnology Inc., Beijing, People's Republic of China). Cells with phorbol-12-myristate-13-acetate $(100 \mathrm{ng} / \mathrm{mL})$ were seeded into 96-well plates and incubated for 72 hours at $37^{\circ} \mathrm{C}$ in the dark, followed by being subjected to different experimental treatments: 1) the cells were incubated with different HY concentrations $(0-1.0 \mu \mathrm{g} / \mathrm{mL})$ for 4 hours at $37^{\circ} \mathrm{C}$ in the dark; 2) the cells were incubated with $0.25 \mu \mathrm{g} / \mathrm{mL}$ HY for $0-4$ hours; 3 ) the cells were exposed to ultrasound for 0-15 minutes; 4) the cells were incubated with different HY concentrations $(0-0.25 \mu \mathrm{g} / \mathrm{mL})$ for 4 hours and exposed to ultrasound for 15 minutes; 5) the cells treated were incubated with $0.25 \mu \mathrm{g} / \mathrm{mL} \mathrm{HY}$ for 4 hours and exposed to ultrasound for $0-15$ minutes; 6) the cells were incubated with $0.25 \mu \mathrm{g} / \mathrm{mL}$ HY for 0-6 hours, followed by 15 minutes of ultrasound irradiation; 7) the cells were incubated with $0.25 \mu \mathrm{g} / \mathrm{mL}$ concentrations of HY, emodin, curcumin, and ALA for 4 hours, then treated with ultrasound for 15 minutes; 8) the cells were incubated with $0.25 \mu \mathrm{g} / \mathrm{mL} \mathrm{HY}$ with or without 0.5 $\mu \mathrm{M}$ CsA (mPTP inhibitor), 1.0 mM NAC (ROS scavenger), $10 \mathrm{mM} \mathrm{NaN}_{3}$ (singlet oxygen scavenger), $100 \mathrm{mM}$ mannitol (hydroxyl radical scavenger), $100 \mu \mathrm{g} / \mathrm{mL}$ SOD (superoxide anion radical scavenger), $200 \mathrm{U} / \mathrm{mL}$ CAT (hydrogen peroxide scavenger), or $20 \mu \mathrm{M} \mathrm{z-VAD}$ (caspase inhibitor) for 4 hours. After a 6-hour incubation following SDT, the medium was removed, and $100 \mu \mathrm{L}$ of medium containing CCK-8 (split ratio 10:1) without FBS was added to the cells, which were then incubated for 1 hour at $37^{\circ} \mathrm{C}$ in the dark. Absorption at 450 $\mathrm{nm}$ was subsequently measured on a microplate reader (Varian Australia Pty Ltd, Melbourne, VIC, Australia). The presented data represent the averages for six wells for each treatment. Each experiment was repeated three times individually.

\section{Cellular uptake of $\mathrm{HY}$}

The cells were incubated with $0.25 \mu \mathrm{g} / \mathrm{mL}$ HY for $0-6$ hours at $37^{\circ} \mathrm{C}$ in the dark. After repeated washes with PBS, intracellular HY was identified using confocal laser scanning microscopy (CLSM) (LSM 510 Meta; Zeiss, Gottingen, Germany). The HY (555 nm excitation wavelength) fluorescence intensity was analyzed using Zeiss CLSM software (ZEN 2009 Light Edition).

\section{Colocalization of $\mathrm{HY}$ with nuclei in THP-I macrophages}

The cells were incubated with $0,0.1,0.2$, or $0.25 \mu \mathrm{g} / \mathrm{mL} \mathrm{HY}$ for 4 hours at $37^{\circ} \mathrm{C}$ in the dark. Then, they were washed twice with PBS and fixed with 4\% paraformaldehyde for 5 minutes before staining with $1.0 \mathrm{~mL}$ of $1.0 \mu \mathrm{g} / \mathrm{mL} \mathrm{4}$, 6-diamidine$2^{\prime}$-phenylindole dihydrochloride (DAPI, F. Hoffmann-La Roche Ltd, Basel, Switzerland) for 5 minutes at $37^{\circ} \mathrm{C}$ in the dark. The colocalization of HY and nuclei was subsequently detected using CLSM. The fluorescence intensity of HY and nuclei (340 nm excitation wavelength) was analyzed with Zeiss CLSM software. 


\section{Colocalization of HY with different organelles in THP-I macrophages}

The cells were incubated with 0 or $0.25 \mu \mathrm{g} / \mathrm{mL}$ HY for 4 hours at $37^{\circ} \mathrm{C}$ in the dark. After repeated washes with PBS, the cells were stained with $0.25 \mu \mathrm{M}$ Mito-Tracker Green (Beyotime Biotechnology Inc.), $0.2 \mu \mathrm{M}$ ER-Tracker Green, $5 \mu \mathrm{M}$ GolgiTracker Green, and 0.4 $\mu$ M Lyso-Tracker Green (ER, Golgi, and lysosome probes from Invitrogen, Eugene, OR, USA) for 20 minutes. After repeated washes with PBS and fixation in 4\% paraformaldehyde for 5 minutes, the cells were stained with $1.0 \mu \mathrm{g} / \mathrm{mL}$ DAPI for 5 minutes to visualize the nuclei. The natural fluorescence of HY was used to visualize its presence in the cells. The fluorescence signals of MitoTracker Green, ER-Tracker Green, Golgi-Tracker Green, and Lyso-Tracker Green (490 nm excitation wavelength) were used to image the mitochondria, endoplasmic reticulum (ER), Golgi apparatus, and lysosomes, respectively, via CLSM. The observed fluorescence intensities were analyzed with Zeiss CLSM software.

\section{Hoechst 33258 and propidium iodide assay}

Hoechst 33258 and propidium iodide (PI) staining was used to detect the apoptosis and necrosis of macrophages. Six hours after SDT, the cells were stained with Hoechst 33258 and PI according to the manufacturer's protocol after repeat washes with PBS. Briefly, macrophages were first incubated with $10 \mu \mathrm{g} / \mathrm{mL}$ PI for 10 minutes at $37^{\circ} \mathrm{C}$ in the dark and then with $5 \mu \mathrm{g} / \mathrm{mL}$ Hoechst 33258 for 5 minutes. After repeated washes with PBS, the cells were examined under a fluorescence microscope (Olympus IX81, Japan) using a filter with a 330-385 nm excitation wavelength and a 420-480 nm emission wavelength. The obtained images were subsequently processed using Image-Pro Plus software (Media Cybernetics, Rockville, MD, USA). The fraction of apoptosis or necrosis among the THP-1 macrophages was calculated in 18 random images.

\section{Transmission electron microscopy examination}

The cells were trypsinized, harvested via centrifugation, and fixed with 2.5\% glutaraldehyde in PBS at 6 hours after SDT. The treated cells were postfixed in $1 \%$ osmium tetroxide $\left(\mathrm{OsO}_{4}\right)$, washed with PBS, and dehydrated in a graded series of ethanol solutions, finishing with isoamyl acetate. The cells were microsectioned into ultrathin sections, stained with uranyl acetate and lead citrate, and then examined under a transmission electron microscope (JEM-1220, JEOL, Tokyo, Japan).

\section{Measurement of intracellular ROS}

The fluorescence intensity of 2', $7^{\prime}$-dichlorofluorescein (DCF; Molecular Probes, Eugene, OR, USA) was used to determine intracellular ROS generation. Within the cell, esterases cleaved the acetate groups on $2^{\prime}, 7^{\prime}$-dichlorofluorescein diacetate (DCFH-DA; Applygen Technologies Co., Ltd, Beijing, People's Republic of China), intracellularly trapping the reduced probe dichlorodihydrofluorescein (DCFH). ROS in the cells would then rapidly oxidize DCFH, yielding the highly fluorescent product DCF. Six hours after SDT, the cells were washed twice with PBS and incubated with DCFH-DA ( $20 \mu \mathrm{M}$, diluted in serum-free medium) for 30 minutes at $37^{\circ} \mathrm{C}$ in the dark. Then, the cells were carefully washed twice with PBS. Immediately after washing, fluorescence was measured via CLSM at $488 \mathrm{~nm}$ excitation and $525 \mathrm{~nm}$ emission wavelengths. The obtained images were subsequently processed using Image-Pro Plus software and Zeiss CLSM software.

\section{Determination of lipoprotein oxidation}

Malondialdehyde (MDA), a stable end product of fatty acid peroxidation, can react with thiobarbituric acid (TBA) under acidic conditions to form an MDA-TBA adduct with a maximum absorbance at $532 \mathrm{~nm}$. Solutions of $1.0 \mathrm{mM} \mathrm{NAC}$ and $10 \mathrm{mM} \mathrm{NaN}_{3}$ were added to confirm the relationship between ROS and MDA. A total of $1.0 \times 10^{5}$ cells were collected, and the mitochondria were isolated after each treatment. Following the manufacturer's instructions for the MDA detection kit (Jiancheng Bioengineering Institute, Nanjing, People's Republic of China), the lipoprotein oxidation level was determined using a microplate reader.

\section{Measurement of the mitochondrial membrane potential}

The fluorescent, lipophilic, cationic probe jc-1 (Beyotime Biotechnology Inc.) was used to assess the mitochondrial membrane potential $(\Delta \Psi \mathrm{m})$. Six hours post-HY-SDT, macrophages were incubated with $10 \mathrm{mg} / \mathrm{mL} \mathrm{jc-1}$ for 20 minutes at $37^{\circ} \mathrm{C}$ away from light. Then, the cells were carefully washed twice with PBS. Immediately after washing, fluorescence was monitored via a fluorescence microscopy. Red-orange fluorescence produced by jc-1 aggregation in the mitochondrial matrix suggested a high $\Delta \Psi \mathrm{m}$. Green fluorescence generated by jc-1 monomers in the cytosol indicated $\Delta \Psi \mathrm{m}$ collapse. The fluorescence intensity was measured at $488 \mathrm{~nm}$ excitation and $530 \mathrm{~nm}$ and $590 \mathrm{~nm}$ emission wavelengths with a fluorospectrophotometer (Varian Australia Pty Ltd). To investigate the relationship between mitochondria, ROS, and singlet oxygen, 
the cells were pretreated with $0.5 \mu \mathrm{M}$ CsA, $1.0 \mathrm{mM}$ NAC, or $10 \mathrm{mM} \mathrm{NaN}_{3}$. The obtained images were subsequently processed using Image-Pro Plus software.

\section{Measurement of changes in the mPTP}

Mitochondria were stained with the acetomethoxy derivate of calcein (calcein-AM) through $\mathrm{Co}^{2+}$ quenching of cytosolic calcein fluorescence. Six hours after SDT, macrophages were loaded with $5 \mu \mathrm{M}$ calcein-AM (GenMed Scientifics Inc., Wilmington, DE, USA) in the presence of $5 \mathrm{mM}$ cobalt chloride in the dark for 20 minutes at $37^{\circ} \mathrm{C}$ as previously described..$^{29}$ The cells were carefully washed twice with PBS, and fluorescence was monitored via fluorescence microscopy. Calcein-AM fluorescence was measured at $525 \mathrm{~nm}$ following excitation at $488 \mathrm{~nm}$. To investigate the mechanism of mPTP opening, the cells were pretreated with NAC and inhibitors of the different subunits (BA, DIDS, and CsA) detected through CLSM. Images were subsequently processed using Image-Pro Plus software and Zeiss CLSM software.

\section{Extraction of mitochondrial and cytosolic fractions}

Mitochondrial and cytosolic proteins were isolated using a mitochondria/cytosol fractionation kit (Beyotime Biotechnology, Inc.) according to the manufacturer's instructions. Briefly, cells were harvested, washed twice with PBS, incubated with ice-cold cytosolic separation buffer containing protease inhibitors, agitated for 30 seconds four times and lysed on ice for 15 minutes. Then, the mixture was centrifuged at 3,000 rpm for 15 minutes at $4^{\circ} \mathrm{C}$. Next, the supernatant was collected and centrifuged at $12,000 \mathrm{rpm}$ for 30 minutes at $4^{\circ} \mathrm{C}$ to obtain a pellet of mitochondria and a cytosolic supernatant. The pellet was incubated with mitochondrial separation buffer for 30 minutes on ice and then centrifuged at 13,000 rpm for 10 minutes at $4^{\circ} \mathrm{C}$ to obtain the mitochondrial fractions. The mitochondrial and cytosolic extracts were used for analyses of BAX and cytochrome $\mathrm{C}$ by Western blotting.

\section{Western blotting analysis}

Sodium dodecyl sulfate-polyacrylamide gel electrophoresis (SDS-PAGE) was performed to analyze the expression of the proapoptotic factor BAX, the antiapoptotic factor Bcl-2, and apoptosis-related proteins, including cytochrome $\mathrm{C}$, cleaved caspase-9, cleaved caspase-3, and cleaved poly(ADP-ribose) polymerase (PARP). To determine the total protein fraction, after 6 hours of incubation following SDT, the cells were washed twice with PBS and lysed with RIPA buffer ${ }^{19}$ on ice for 30 minutes. Then, the cells were harvested and collected via centrifugation at $12,000 \mathrm{rpm}$ for 30 minutes at $4^{\circ} \mathrm{C}$. The bicinchoninic acid protein assay reagent (Beyotime Biotechnology, Inc.) was used to measure the protein concentration in the lysate. Protein samples were mixed with $5 \times$ sample loading buffer (10\% SDS, 5\% $\beta$-mercaptoethanol, 15\% glycerol, $0.01 \%$ bromophenol blue, $200 \mathrm{mM}$ Tris- $\mathrm{HCl}, \mathrm{pH}$ 6.7) and separated on a $10 \%-15 \%$ linear SDS-PAGE gel, then transferred to polyvinylidene fluoride membranes (Millipore, MA, USA). Then, the polyvinylidene fluoride membranes were blocked with $5 \%$ nonfat milk in Tris-buffered saline-Tween $20(0.05 \%)$ at room temperature for 2 hours. The membranes were subsequently probed with primary antibodies (against BAX, Bcl-2, cytochrome C, cleaved caspase-9, cleaved caspase-3, and cleaved PARP; Cell Signaling Technology, Inc., Danvers, MA, USA) overnight at $4^{\circ} \mathrm{C}$. All primary antibodies were diluted 1:1,000. After three washes with Trisbuffered saline-Tween 20, the membranes were incubated with alkaline phosphatase-labeled secondary antibodies at room temperature for 1 hour. The resultant immune complexes were detected with enhanced chemiluminescence reagents. All secondary antibodies were diluted 1:1,000. Anti- $\beta$-actin (Cell Signaling Technology, Inc.) was used to confirm equal cytosol loading. Anti-Hsp 60 (Cell Signaling Technology, Inc.) was employed to confirm equal mitochondria loading. The percent increases or decreases of protein content were estimated compared with the vehicle control (100\%).

\section{Statistical analysis}

All values were expressed as the means \pm standard deviation of at least three independent experiments. Differences between groups were subjected to one-way analyses of variance. $P<0.05$ was considered significant.

\section{Results Physical optics characterization of HY}

The structure of HY is shown in Figure 2A. The absorption spectra of HY occur at $555 \mathrm{~nm}$ and $600 \mathrm{~nm}$. The emission spectrum of HY occurs at $647 \mathrm{~nm}$.

\section{Cell viability after different treatments}

The CCK-8 assay indicated that cell viability was influenced by the applied HY concentrations and ultrasound exposure times (Figure 2C). Cell viability declined with increasing HY concentrations and was significantly reduced at a concentration of $0.3 \mu \mathrm{g} / \mathrm{mL}$. Cell viability remained stable in the cultures treated with $0.25 \mu \mathrm{g} / \mathrm{mL} H Y$ with increasing incubation times. There was no observed difference between the cell viabilities of cultures exposed to ultrasound for $0,1,3,5,10$, and 15 minutes. 
Among the cultures treated with different HY concentrations for the same 15-minute duration of ultrasound irradiation, cell viability decreased with an increasing drug concentration. When the cells were incubated with $0.25 \mu \mathrm{g} / \mathrm{mL} \mathrm{HY}$ and subjected to different durations of ultrasound irradiation, cell viability decreased with increasing time. Hence, the application of $0.25 \mu \mathrm{g} / \mathrm{mL} \mathrm{HY}$ and 15-minute ultrasound exposure represented the optimal conditions for SDT to maximize the decrease in cell viability. Figure $2 \mathrm{~B}$ shows that when cells were irradiated for 15 minutes, cell viability decreased with an increasing incubation time of cells loaded with $0.25 \mu \mathrm{g} / \mathrm{mL}$ HY, with no significant difference being observed from 4 to 6 hours. Figure 2D shows that the cell viabilities of cultures treated with emodin, curcumin, ALA, and HY alone did not change. However, cell viability was decreased in the HY-SDTtreated cultures compared with the other three sonosensitizer groups. Figure 3E shows that the broad-spectrum caspase inhibitor z-VAD was able to prevent the death of macrophages caused by HY-SDT. Figure 4A indicates that mannitol, SOD, $\mathrm{CAT}$, and $\mathrm{NaN}_{3}$ alone did not induce a decrease in cell viability; instead, they were observed to inhibit cell death. $\mathrm{NaN}_{3}$ had the most significant effect, which indicated that singlet oxygen was a major component of the ROS in the cells. Figure 4B shows that NAC inhibited the decrease in cell viability without eliciting any cytotoxicity.

\section{Intracellular accumulation of HY in THP-I macrophages}

To study the intracellular metabolic kinetics of HY, the mean fluorescence intensity of HY in THP-1 macrophages was detected via CLSM after incubation with $0.25 \mu \mathrm{g} / \mathrm{mL} \mathrm{HY}$ for different times. The initial fluorescence intensity of HY was less obvious at 30 minutes (Figure 2B). From 1 hour to 6 hours, fluorescence initially gradually increased over time, peaked at 4 hours, and then faded.

\section{Colocalization of HY with nuclei in THP-I macrophages}

To further determine the localization of HY to the nuclei and the influence of different HY concentrations on the relationship between HY and nuclei, we labeled both HY and nuclei in THP-1 macrophages. The intracellular accumulation of HY increased with higher HY treatment concentrations. The colocalization between HY and nuclei is shown as bright pink areas in overlaid images of cells treated with $0.1,0.2$, and $0.25 \mu \mathrm{g} / \mathrm{mL} \mathrm{HY}$. In general, the greater the HY

A<smiles></smiles>
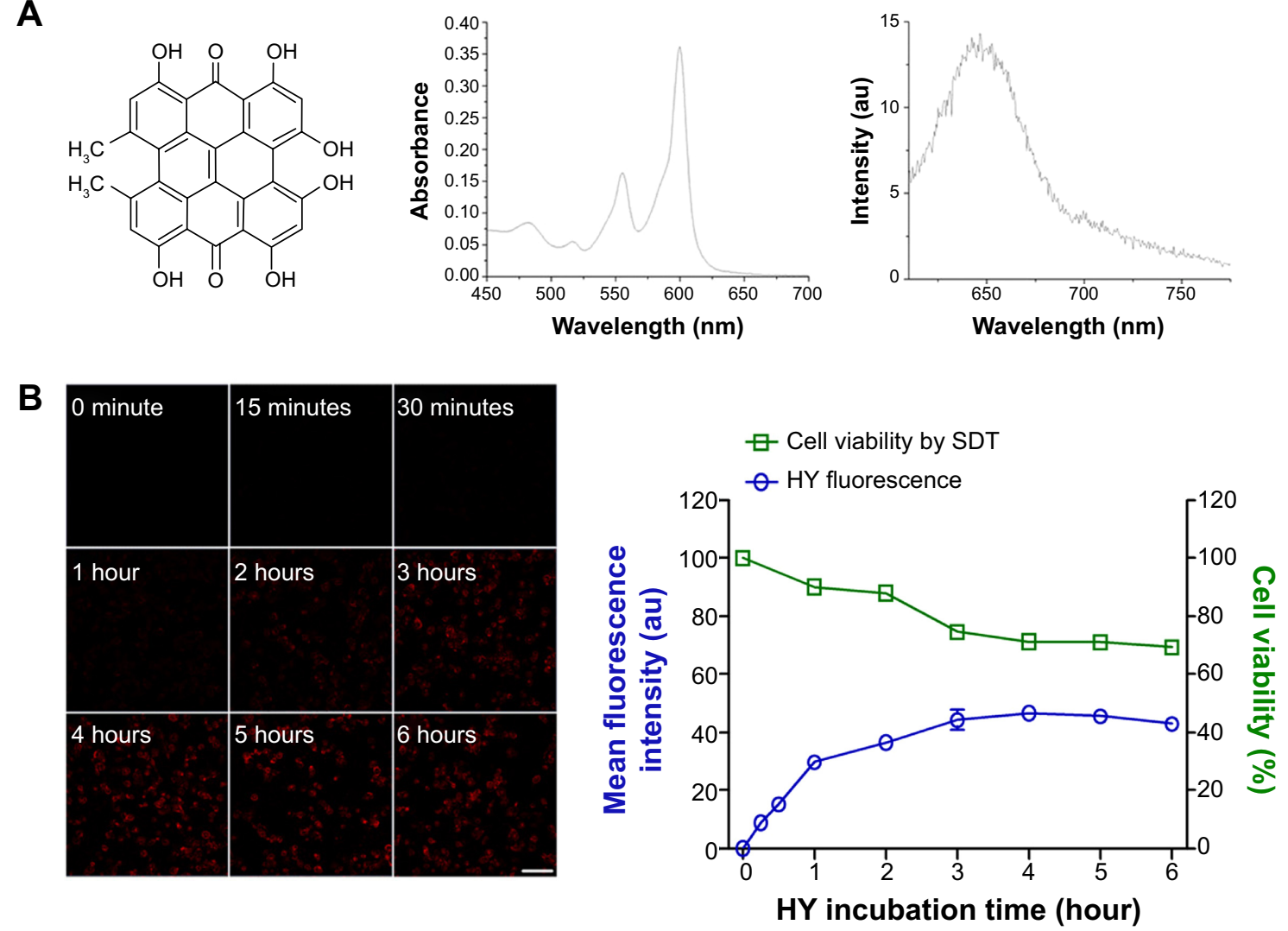

Figure 2 (Continued) 
C
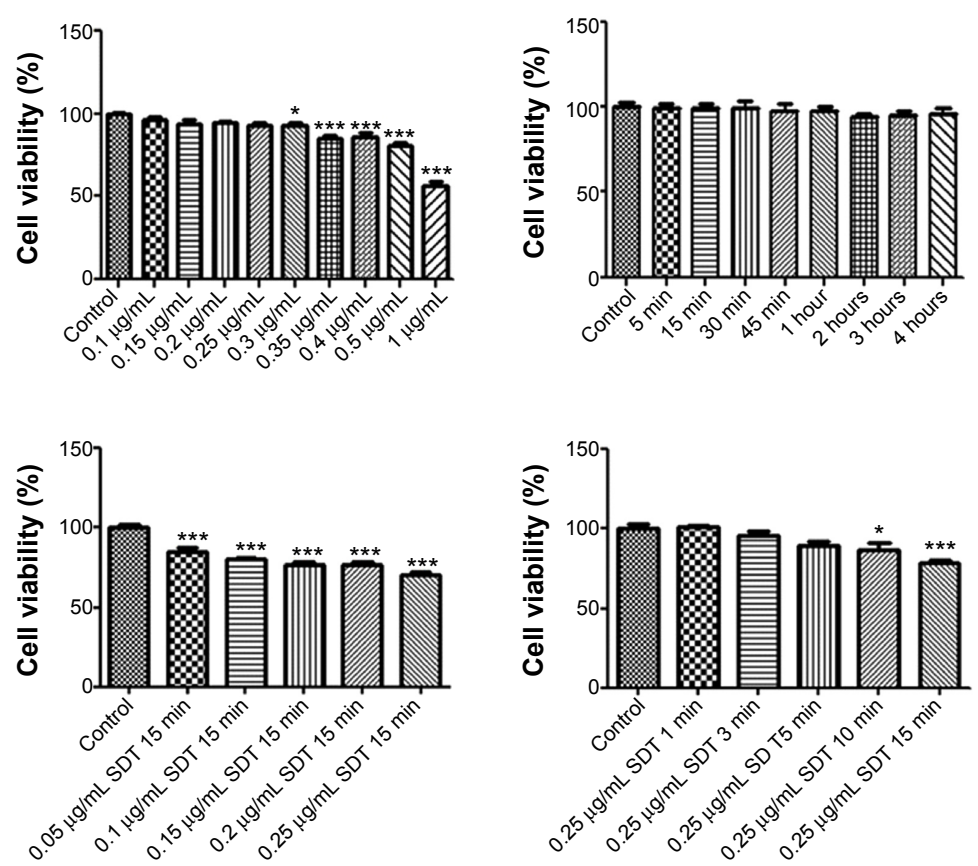
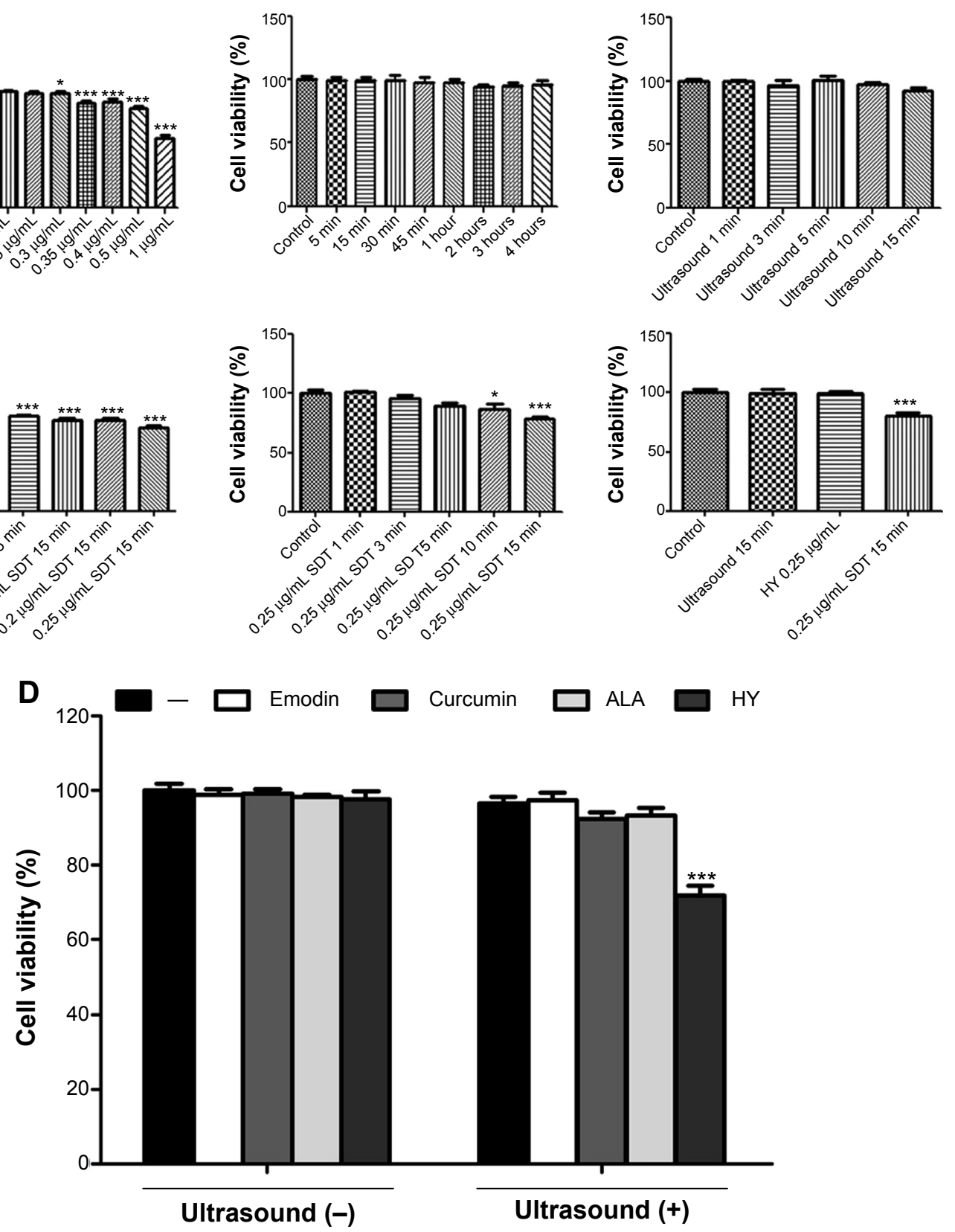

Figure 2 The sonosensitizer hypericin (HY) and the effect induced by HY-sonodynamic therapy (SDT).

Notes: (A) The structure of HY (left). The absorption and emission spectra of HY (right). (B) Intracellular accumulation of HY in THP-I macrophages (scale bar, 0.I mm). (C) Cell viability after different treatments assessed in a CCK-8 assay. (D) The cell viability of THP-I macrophages induced by SDT mediated by different sonosensitizers. The macrophages were treated with emodin $(0.25 \mu \mathrm{g} / \mathrm{mL})$, curcumin $(0.25 \mu \mathrm{g} / \mathrm{mL})$, 5-aminolevolinic acid $(\mathrm{ALA})(0.25 \mu \mathrm{g} / \mathrm{mL})$, or $\mathrm{HY}(0.25 \mu \mathrm{g} / \mathrm{mL})$. $* P<0.05$, $* * * P<0.00 \mathrm{I}$ compared with the control group.

concentration, the higher the pink intensity (Figure 5A). The results indicated that HY remained primarily in the cytosol and only partially localized to the nucleus.

\section{Colocalization of HY with different organelles in THP-I macrophages}

HY was observed to migrate into mitochondria. The colocalization between HY and mitochondria is shown as yellow areas in overlaid images of cells containing $0.25 \mu \mathrm{g} / \mathrm{mL}$ HY (Figure 5B). The results revealed that HY was primarily localized to the mitochondria. To further assess the localization of $\mathrm{HY}$ in different organelles that might represent the key site responsible for the effect of HY-SDT, we loaded the cells with Mito-Tracker Green, ER-Tracker Green, Golgi-Tracker Green, and Lyso-Tracker Green (shown in Figure 6). The pattern of HY (shown in red) corresponded well with those 

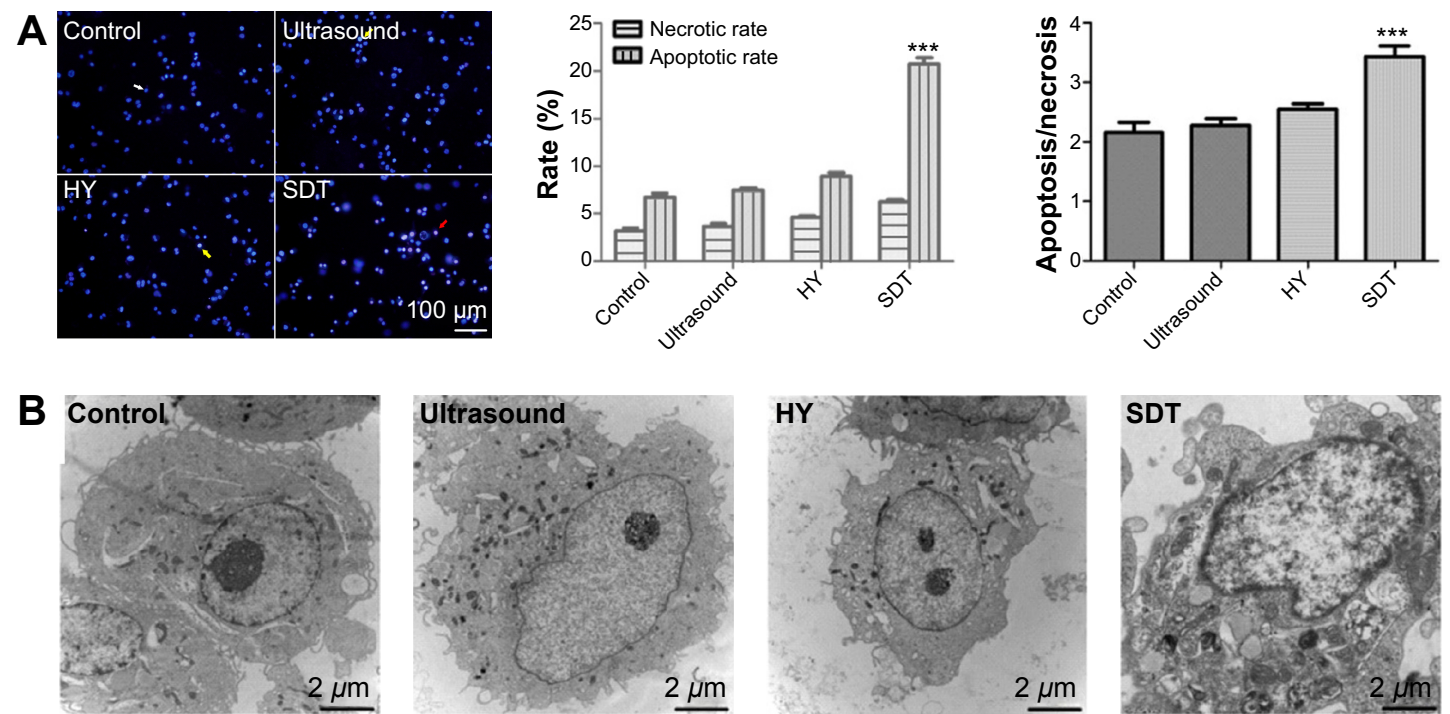

C
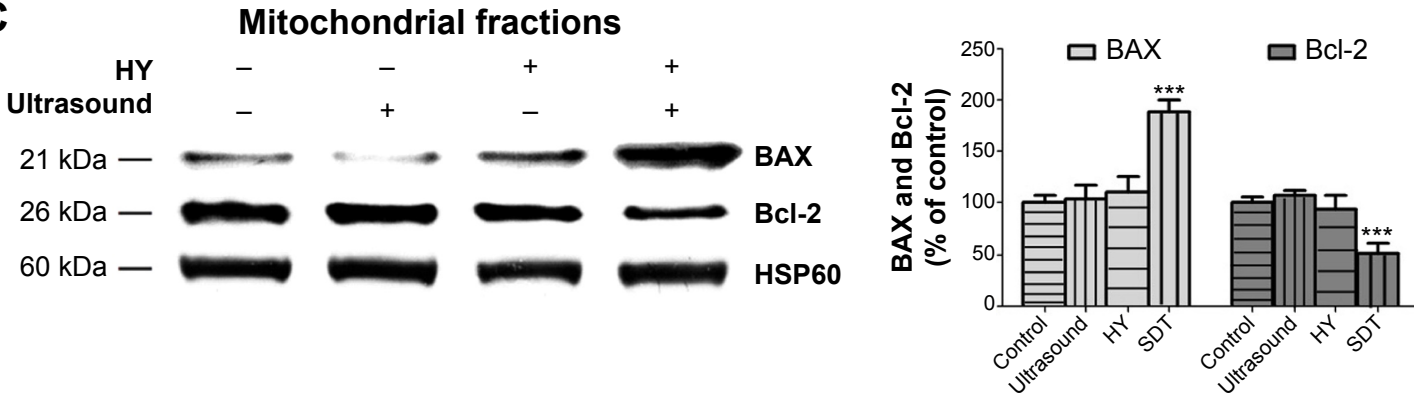

D
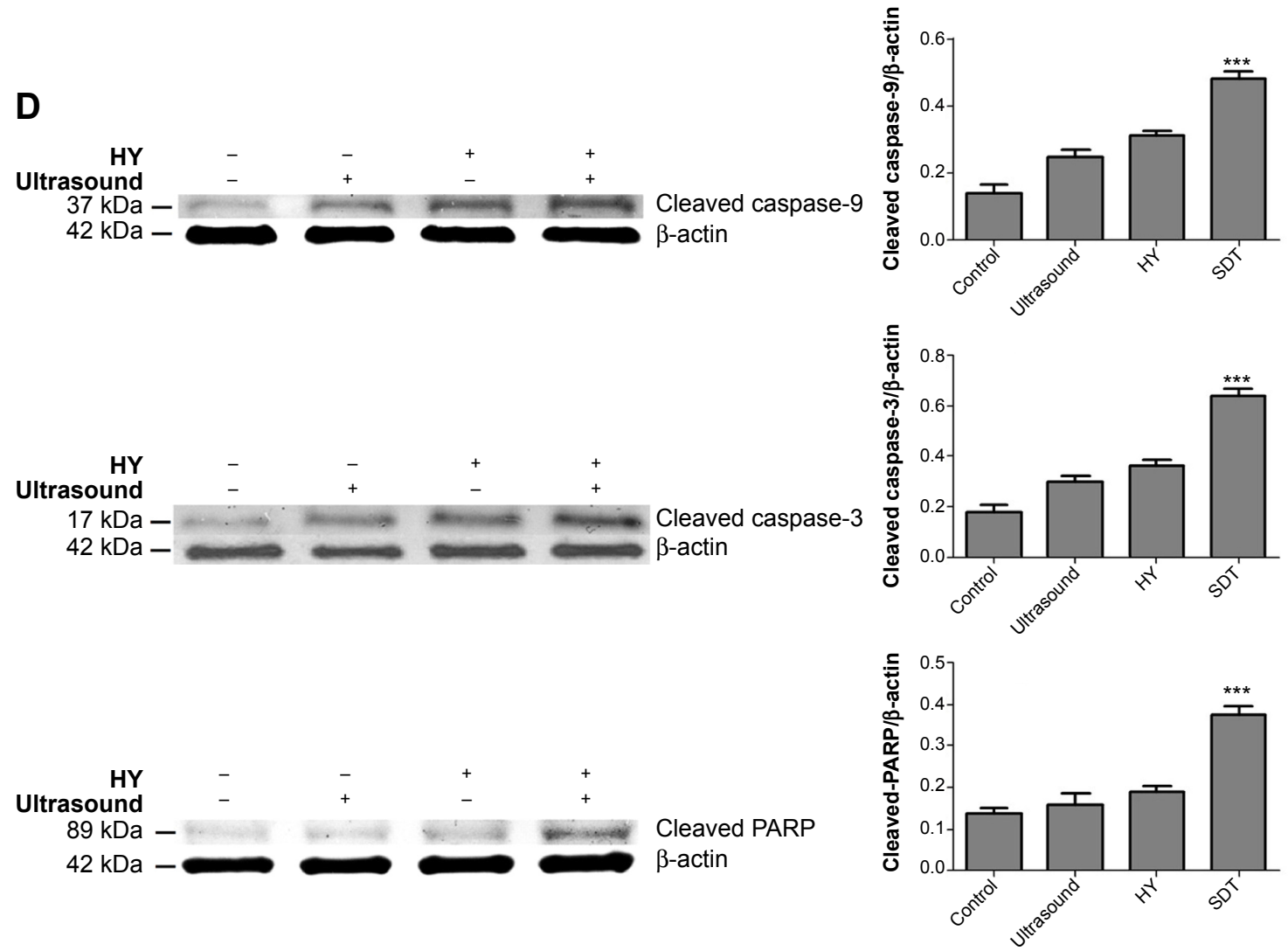

Figure 3 (Continued) 


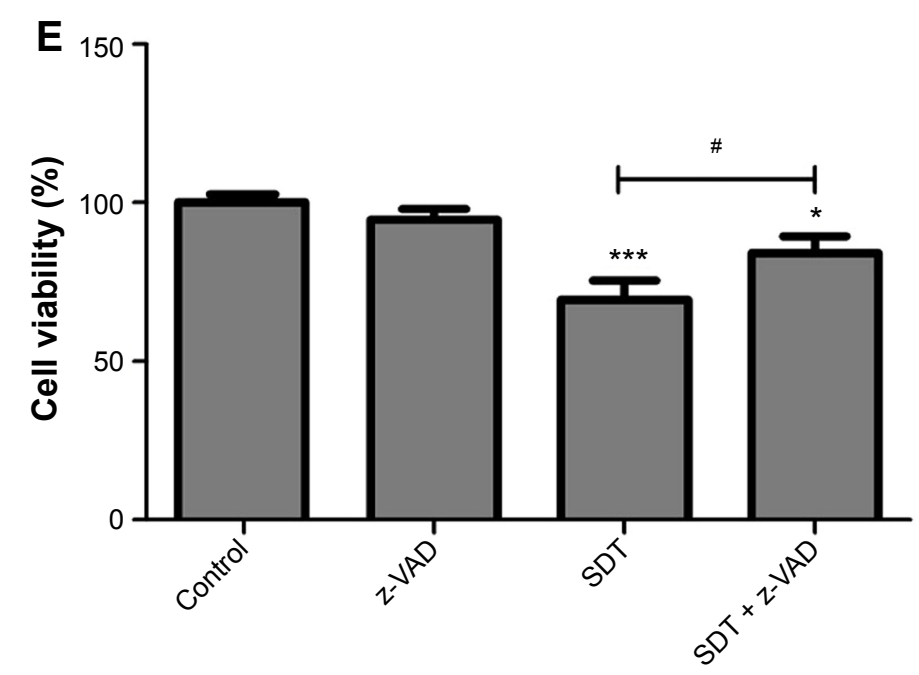

Figure 3 Hypericin (HY)-sonodynamic therapy (SDT)-mediated apoptosis in THP-I macrophages.

Notes: (A) The apoptosis and necrosis of THP-I macrophages were detected via Hoechst 33258 and propidium iodide assays. Normal cells are indicated by uniform blue fluorescence (white arrow). Apoptotic cells appear as bright blue fluorescent spots (yellow arrow). Necrotic nuclei are indicated by pink fluorescence (red arrow; scale bar, $0.1 \mathrm{~mm}$ ). The percentage of necrotic and apoptotic THP-I macrophages and the apoptosis/necrosis ratio are shown. (B) Morphological alterations induced by HY-SDT in macrophages. Mitochondrial swelling and the disappearance of cristae (black arrow) could be observed in the cells of the SDT group. (C) Protein levels of the proapoptotic factor BAX and the antiapoptotic factor Bcl-2 in mitochondria. (D) Expression of the apoptosis-related proteins cleaved caspase-3, cleaved caspase-9, and cleaved poly(ADPribose) polymerase (PARP). (E) The inhibitory effects of $z-V A D$ in preventing the decreased cell viability induced by $H Y-S D T$. $* P<0.05$, $* * * P<0.00 \mathrm{I}$ compared with the control, $" P<0.05$ compared with the SDT.

of the organelle probes (shown in green), with a bright yellow color representing the overlapping regions, which indicated that HY could localize in all four types of organelles.

\section{Apoptosis and necrosis of THP-I macrophages}

Fluorescent images showed that in the SDT group there were more bright blue fluorescing cells than pink fluorescing cells, indicating that SDT could induce both apoptosis and necrosis in THP-1 macrophages, though the rate of apoptosis $(20.7 \% \pm 2.5 \%)$ was higher than the rate of necrosis $(6.3 \% \pm 0.8 \%)$. The maximum ratio of apoptosis to necrosis was $3.4 \pm 0.7$ under SDT using $0.25 \mu \mathrm{g} / \mathrm{mL} H Y$ with 15 minutes of ultrasound exposure (Figure 3A).

\section{Transmission electron microscopy examination}

In the SDT group, the cells showed typical apoptotic morphological changes, including the disappearance of cell microvilli, nuclear chromatin condensation on the nuclear envelope, and significant mitochondrial swelling with disappearing crista (Figure 3B). These results suggested that SDT induced apoptosis of THP-1 macrophages.

\section{Measurement of intracellular ROS}

ROS-induced cellular damage is considered to be the main mechanism involved in SDT, ${ }^{21,30,31}$ and the results of this study confirmed ROS generation (Figure 4C). The relative ROS level in the ultrasound alone group was slightly but significantly increased by $129.2 \% \pm 8.8 \%$ in the HY alone group and was increased by $244.8 \% \pm 7.5 \%$ in the SDT group. ROS generation was significantly decreased in the SDT group pretreated with $\mathrm{NAC}$ and $\mathrm{NaN}_{3}$. To further determine which chemical was more efficient in altering SDT-induced apoptosis, several other free radical scavengers, including mannitol, SOD, CAT, and $\mathrm{NaN}_{3}$, were tested before the treatment. The effects of the scavengers on the THP-1 macrophages after 15 minutes of ultrasound exposure are compared in Figure 4A. Cell viability under SDT was significantly increased in the presence of $\mathrm{NaN}_{3}$, but there was no obvious change when cells were pretreated with mannitol, SOD, or $\mathrm{CAT}$, indicating that singlet oxygen might be the main factor involved in cell damage post-SDT.

\section{Determination of lipoprotein oxidation}

According to a previous study, the accumulated ROS in tumor cells can cause oxidizing reactions in biomolecules that may disrupt the integrity of lipid membranes and proteins. ${ }^{30} \mathrm{We}$ detected MDA contents and found that in the ultrasound and HY groups, MDA contents were slightly increased compared with the control group (Figure 7E). The MDA content of the SDT group was significantly increased to three times that of the control. However, the MDA content of the SDT group decreased upon pretreatment with $\mathrm{NAC}$ and $\mathrm{NaN}_{3}$. 
A

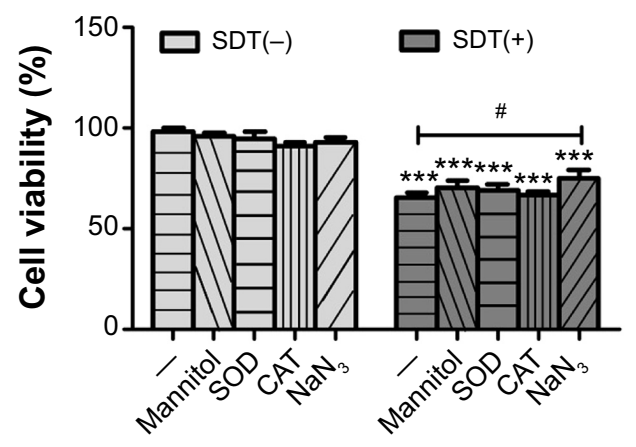

C

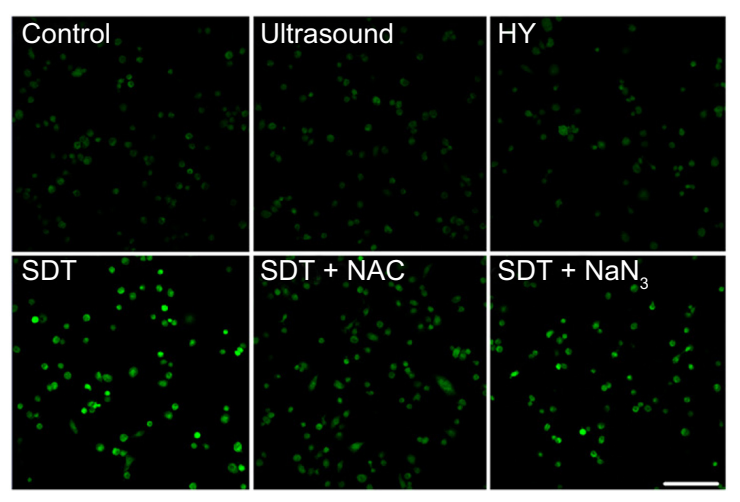

B
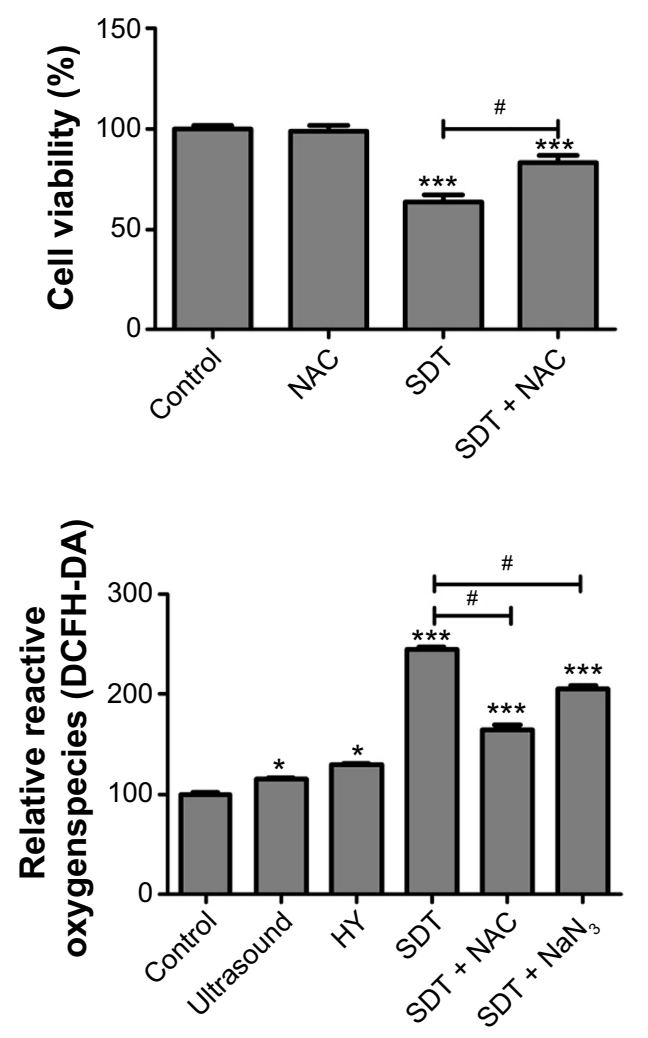

D

\section{Mitochondrial fractions}
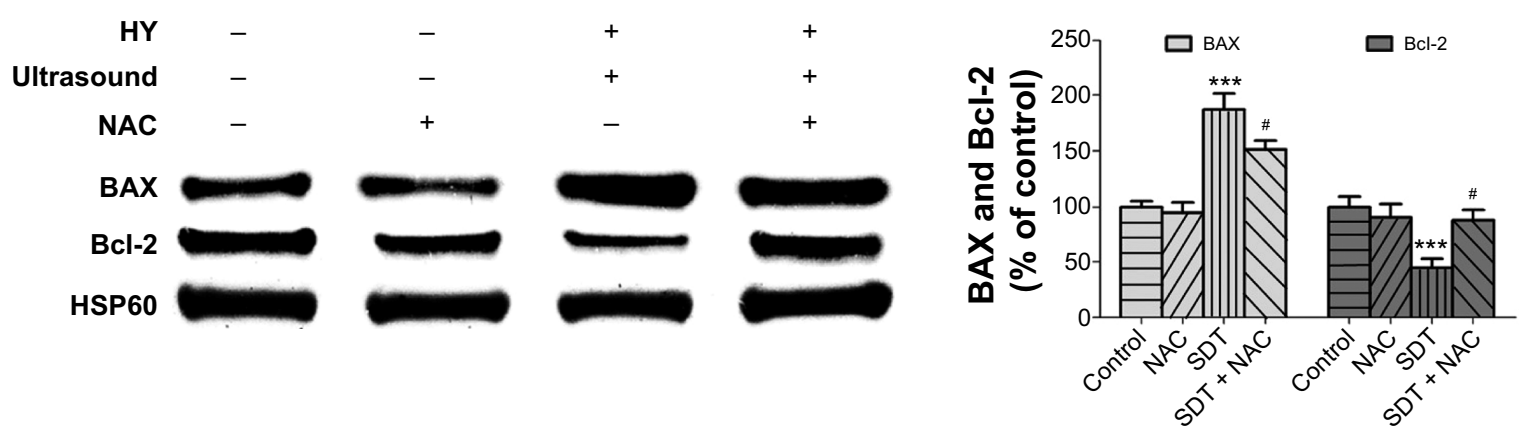

E

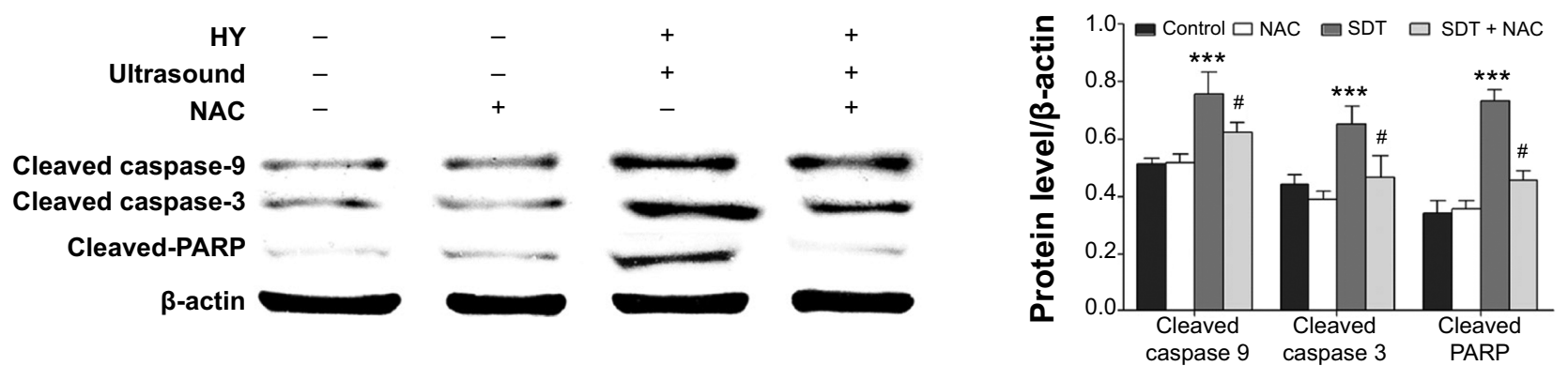

Figure 4 Intracellular reactive oxygen species (ROS) generation in THP-I macrophages induced by hypericin (HY)-sonodynamic therapy (SDT) and the effect of N-acetyl cysteine (NAC) on the expression of apoptosis-related proteins.

Notes: (A) Effects of other several free radical scavengers on cell viability after 15 minutes of ultrasound exposure in the presence of $0.25 \mu \mathrm{g} / \mathrm{mL} \mathrm{HY}$. (B) Cell viability of THP-I macrophages mediated by HY-SDT when pretreated with NAC. (C) The relative fluorescence intensity of ROS generated intracellularly after different treatments (scale bar, $0.1 \mathrm{~mm}$ ). (D) Effect of NAC on the expression of BAX and Bcl-2 in mitochondria. (E) Effect of NAC on the expression of the apoptosis-related proteins cleaved caspase-3, cleaved caspase-9, and cleaved poly(ADP-ribose) polymerase (PARP). $* P<0.05$, $* * * P<0.001$ compared with the control, ${ }^{*} P<0.05$ compared with the SDT. Abbreviation: DCFH-DA, 2',7'-dichlorofluorescein diacetate. 
A

A THP-1 macrophage

DAPI

HY

Overlay

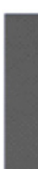

$0.1 \mu \mathrm{g} / \mathrm{mL}$

$0 \mu \mathrm{g} / \mathrm{mL}$
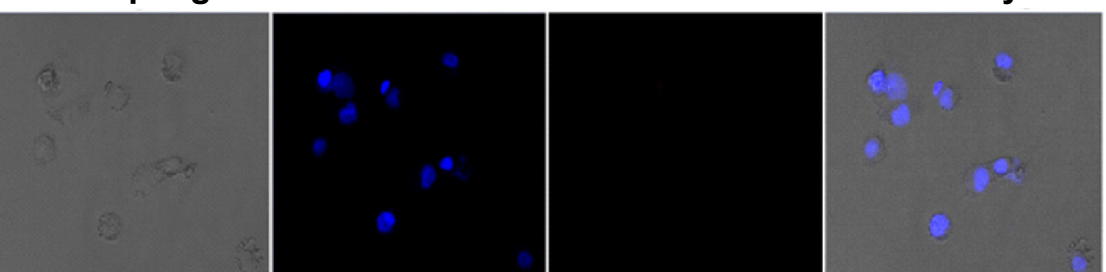

$0.2 \mu \mathrm{g} / \mathrm{mL}$

$0.25 \mu \mathrm{g} / \mathrm{mL}$
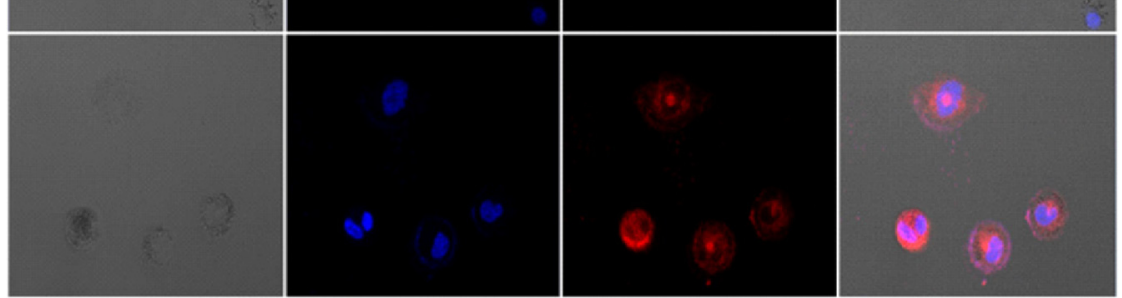

B

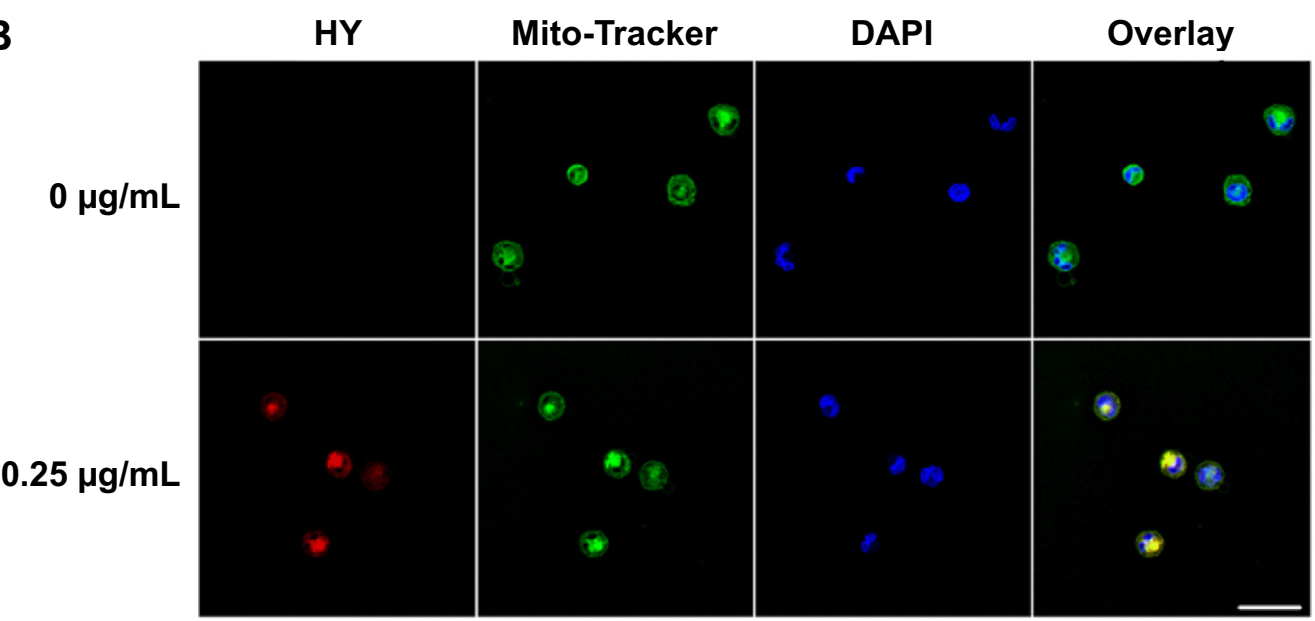

Figure 5 Subcellular localization of hypericin (HY) in THP-I macrophages.

Notes: (A) Colocalization of HY and nuclei. The cells were stained with 4',6-diamidine-2'-phenylindole dihydrochloride (DAPI) to visualize nuclei (shown in blue). The natural fluorescence of HY (shown in red) was used to image its presence in the cells (scale bar, $0.05 \mathrm{~mm}$ ). (B) Colocalization of HY and mitochondria. The cells were stained with Mito-Tracker Green to visualize mitochondria (shown in green) and with DAPI to visualize nuclei (shown in blue; scale bar, $0.05 \mathrm{~mm}$ ).

\section{Detection $\Delta \Psi \mathrm{m}$ loss}

Changes in $\Delta \Psi m$ were analyzed using jc-1, which selectively enters the mitochondria. A reversible color change from red to green indicates a decrease in $\Delta \Psi \mathrm{m} .{ }^{32}$ The release of jc-1 into depolarized mitochondria caused a reduction of the red fluorescence intensity. There was no effect on the intensity of red-orange fluorescence among the cells in the control, ultrasound alone or HY alone groups (Figure 7F). The SDT group exhibited the highest green fluorescence intensity. The red-orange fluorescence intensity decreased, and green fluorescence appeared in a fraction of the SDT group pretreated with $\mathrm{CsA}, \mathrm{NAC}$, and $\mathrm{NaN}_{3}$. The relative $\Delta \Psi \mathrm{m}$ level 


\section{ER-Tracker}

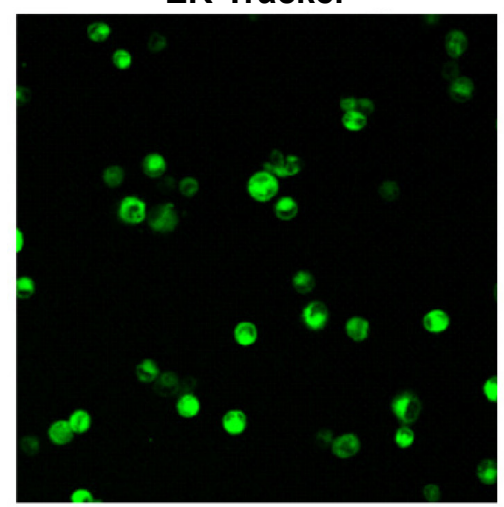

Golgi-Tracker

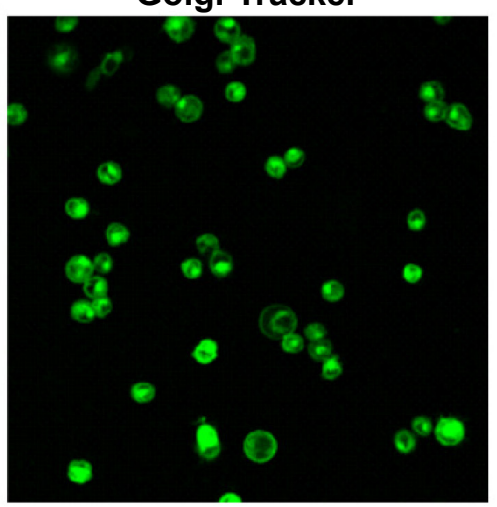

Lyso-Tracker

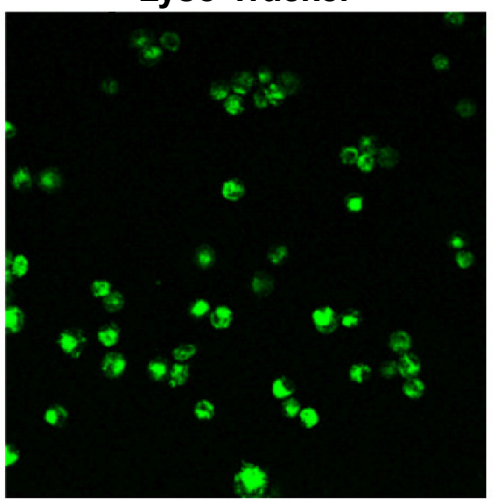

Mito-Tracker

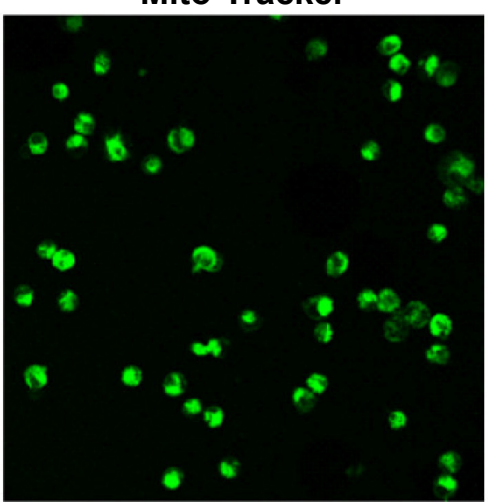

HY

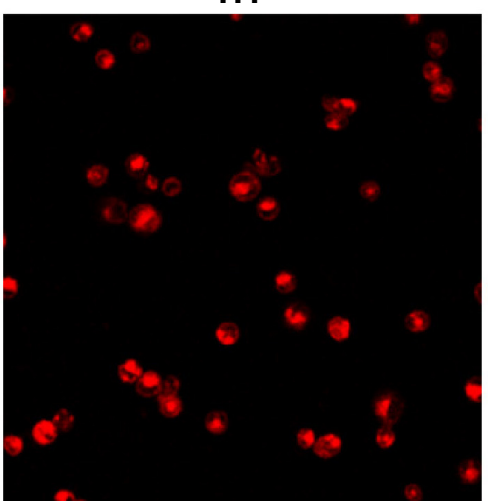

HY

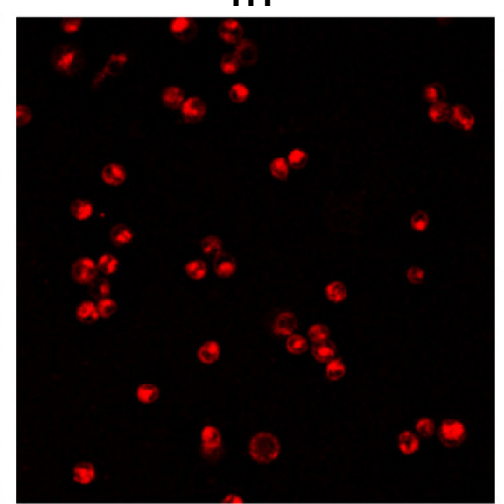

HY

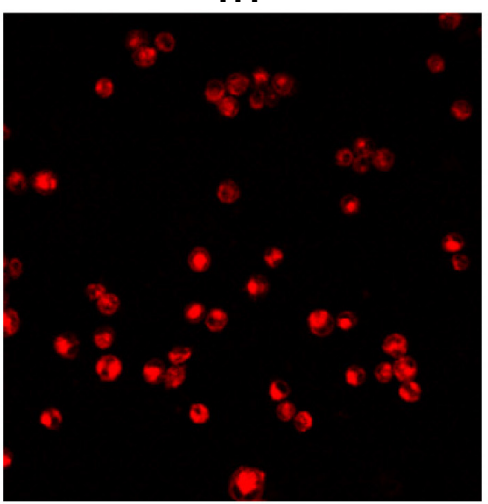

HY

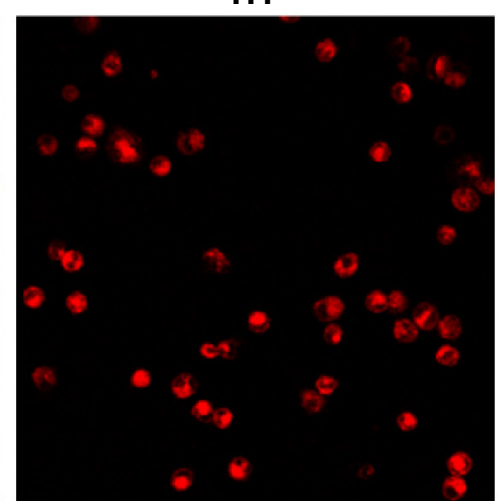

Overlay

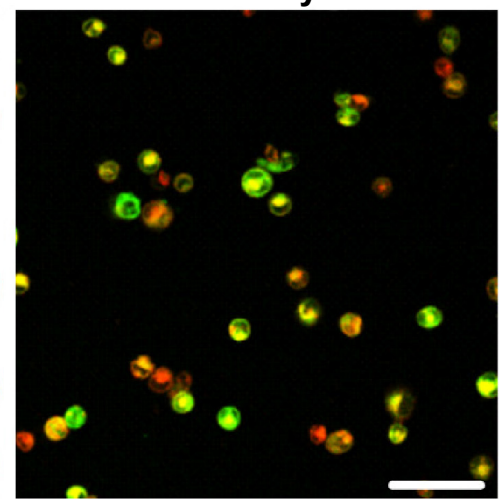

Overlay

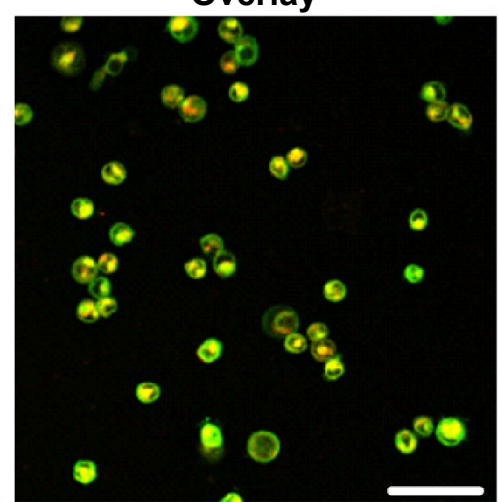

\section{Overlay}

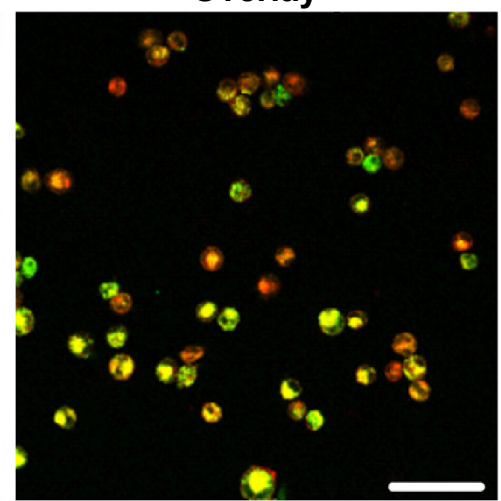

\section{Overlay}

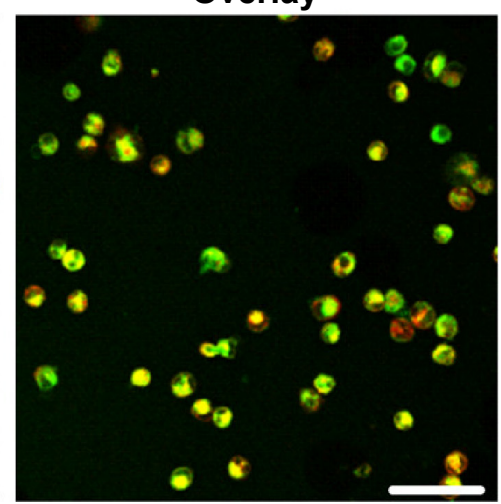

Figure 6 Subcellular localization of hypericin (HY) in THP-I macrophages observed using confocal laser scanning microscopy with specific organelle probes.

Notes: Green represents the four organelle probes; red represents HY; and the merged image of HY and the organelle probes is shown in yellow color (scale bar, $0.05 \mathrm{~mm}$ ). 
in the ultrasound alone group was similar to that of the control. However, the relative $\Delta \Psi \mathrm{m}$ level in the SDT group was reduced to $63.0 \% \pm 3.4 \%$. After pretreatment with CsA, NAC, or $\mathrm{NaN}_{3}$, the relative $\Delta \Psi \mathrm{m}$ level increased to $81.5 \% \pm 4.2 \%$, $87.3 \% \pm 2.8 \%$, or $86.7 \% \pm 6.3 \%$, respectively.

\section{mPTP activity}

To explore whether mPTP participated in the apoptotic mechanism induced by HY-SDT, changes in MPTP were assessed through calcein-AM staining. The green fluorescence intensity decreased in a time-dependent manner (Figure 7D). The relative mPTP fluorescence level in the SDT group decreased with an increasing post-SDT incubation time, remaining unchanged from 4 to 6 hours. To further confirm which of the mPTP subunits presented the main function, we used different inhibitors of the subunits. The results showed that the green fluorescence intensity was lowest in the SDT group and was increased by adding the mPTP inhibitors BA, DIDS, CsA, and NAC (Figure 7C). The relative $\mathrm{mPTP}$ fluorescence level in the SDT group was reduced significantly to $57.8 \% \pm 5.6 \%$. After adding CsA, the fluorescence level increased to $87.1 \% \pm 7.1 \%$ above the intensities in the BA and DIDS groups. From these results, we concluded that HY-SDT could induce MPTP opening and that cyclophilin D represented the main functional site.

\section{Changes in apoptosis-related proteins expression}

To further confirm the role of SDT in inducing apoptosis, we monitored the proapoptotic factor BAX, the antiapoptotic factor Bcl-2, and changes in key apoptosis-related proteins by Western blotting. Compared with the control group, SDT caused a significant increase in mitochondria-localized BAX and a corresponding decrease of BAX in the cytosol (Figure 7A). The opposite pattern was observed for cytochrome $c$, which decreased in mitochondria and increased in the cytosol (Figure 7B). Moreover, the upregulated expression of BAX and the downregulated expression of Bcl-2 increased the ratio of BAX to Bcl-2 (Figure 3C). The expression levels of cleaved caspase-9, cleaved caspase-3, and
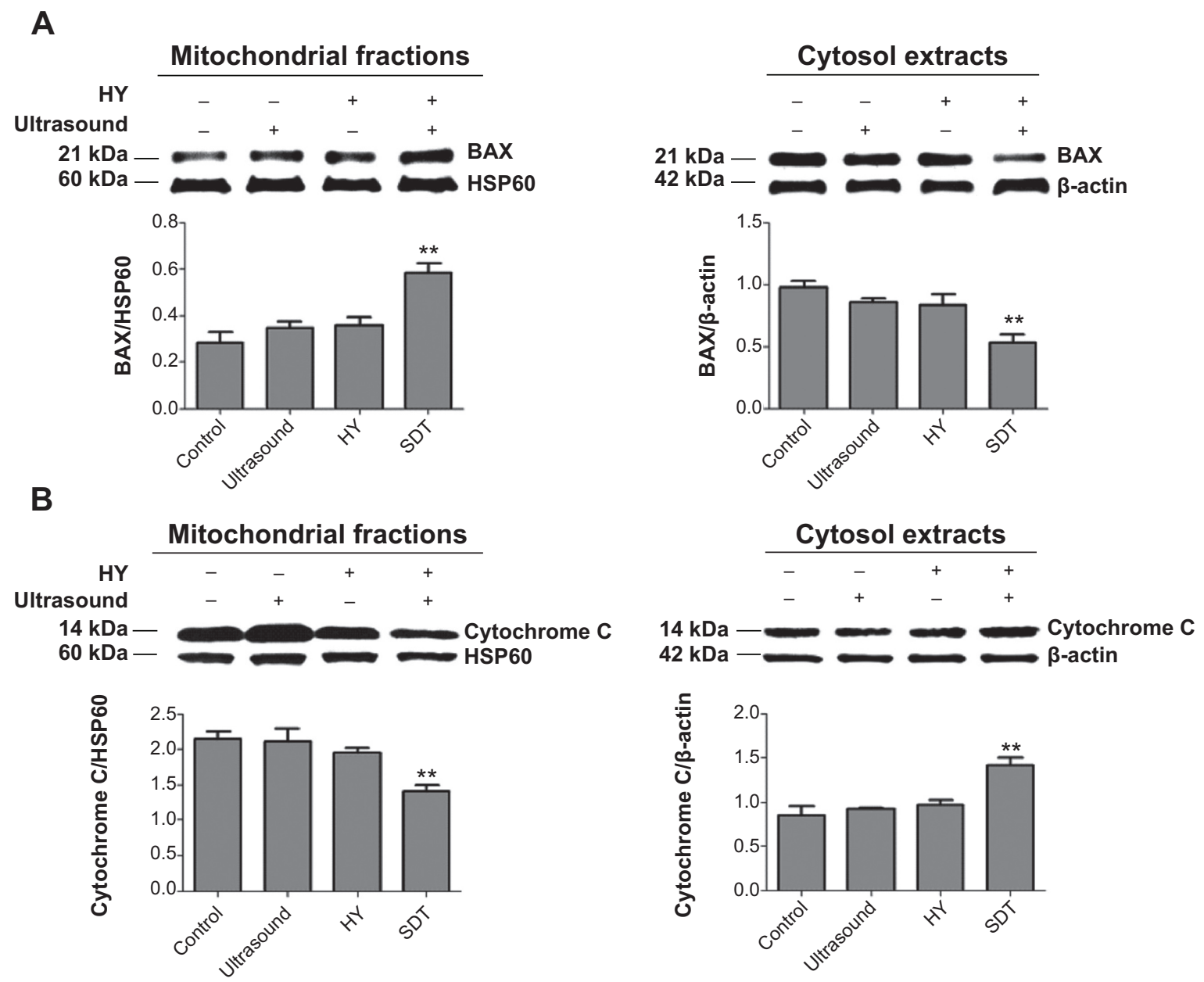

Figure 7 (Continued) 
C

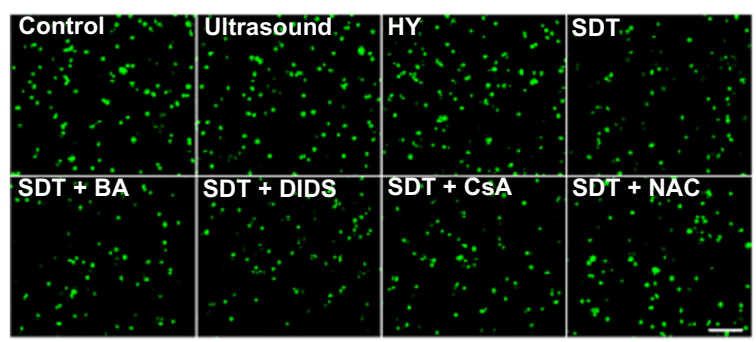

D

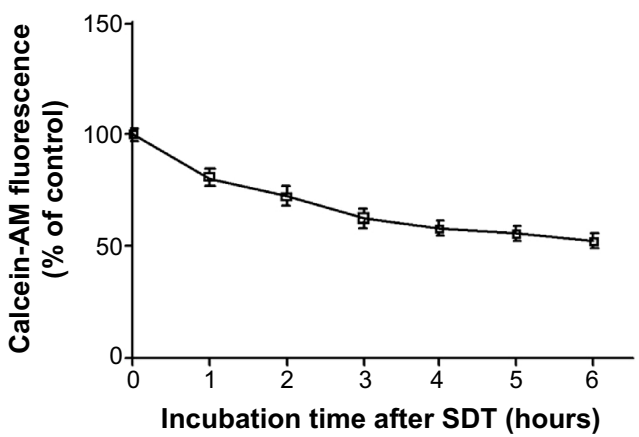

$\mathbf{F}$

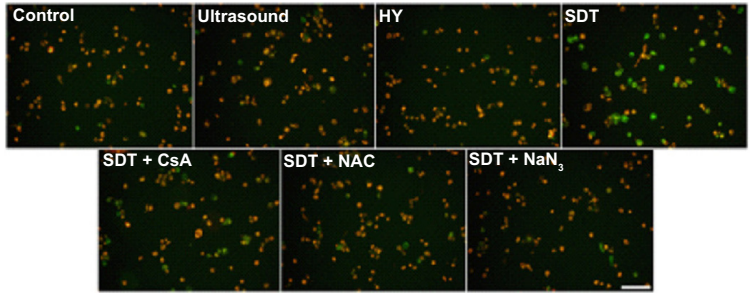

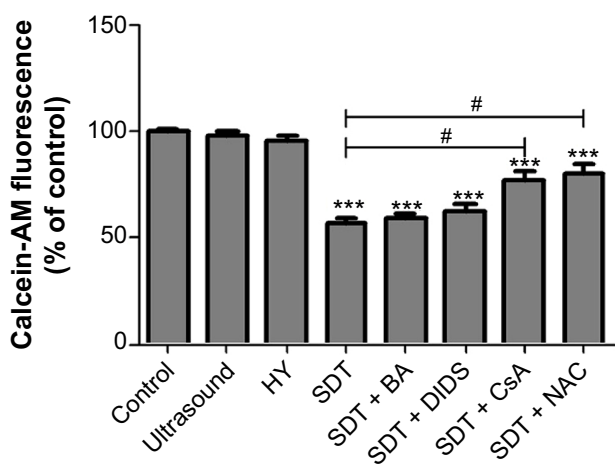

$\mathbf{E}$
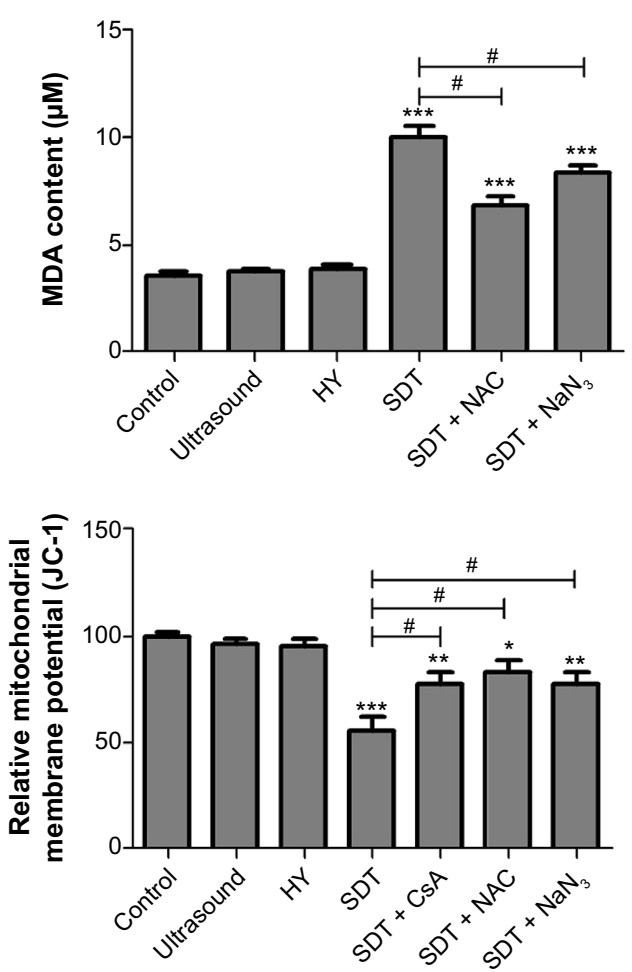

Figure 7 Translocation of BAX and cytochrome $C$ and mitochondrial changes.

Notes: (A) Protein levels of BAX in the mitochondria and cytosol. (B) Protein levels of cytochrome $C$ in the mitochondria and cytosol. (C) Measurement of changes in mitochondrial permeability transition pore (mPTP) in THP-I macrophages pretreated with inhibitors of different subunits. (D) Measurement of changes in mPTP in THP-I macrophages induced by hypericin (HY)-sonodynamic therapy (SDT) after different incubation times. The relative fluorescence intensity of $\mathrm{mPTP}$ was detected via fluorescence microscopy with acetomethoxy derivate of calcein (calcein-AM). (E) Malondialdehyde (MDA) content of mitochondria in THP-I macrophages. (F) Alteration of mitochondrial membrane potential induced by HY-SDT (scale bar, $0.1 \mathrm{~mm}$ ). $* P<0.05$, $* * P<0.01$, *** $P<0.00$ I compared with the control, ${ }^{*} P<0.05$ compared with the SDT group.

Abbreviations: NAC, N-acetyl cysteine; CsA, cyclosporin A; BA, bongkrekic acid; DIDS, 4,4'-diisothiocyanatostilbene-2,2'-disulfonic acid disodium.

cleaved PARP increased upon SDT (Figure 3D). However, treatment with NAC reversed the levels of BAX and Bcl-2 in mitochondria (Figure 4D) and blocked the activation of caspases (Figure 4E).

\section{Discussion}

AS, a chronic inflammatory vascular disease, is characterized by an intimal mass accumulation of macrophages, which are cells that engulf lipids and produce diverse inflammatory mediators. ${ }^{33}$ Moreover, macrophages play a crucial role in the progression and instability of atherosclerotic plaques. ${ }^{34-36}$
Thus, promoting the clearance of macrophages from plaques is an alternative strategy for treating AS. SDT, which combines low-intensity ultrasound with different sonosensitizers, has been demonstrated to induce apoptosis in a variety of cell types relevant to different diseases. In recent years, SDT has attracted more interest as a promising technique for the directed treatment of AS with minimal undesirable damage to surrounding normal tissues. ${ }^{37}$ Most sonosensitizers are derived from photosensitizers, such as HP, protoporphyrin IX, and ALA. As a photosensitizer, HY (whose absorption and emission wavelengths are provided in Figure 2A) has 
been successfully used in PDT of several types of tumor cells. $^{20,23,25,28}$ This article reports the first study to examine the cytotoxic and proapoptotic effects of HY-SDT on THP-1 macrophages and the underlying molecular mechanism. We demonstrated that HY-SDT could significantly induce apoptosis in THP-1 macrophages in vitro. The apoptotic effect of HY-SDT occurred through the mitochondria-caspase pathway involving ROS, which regulated MPTP opening.

Macrophages are functionally active cells in atherosclerotic plaques and can release cytokines to promote the continuous infiltration of macrophages. This process results in lipoprotein accumulation and free cholesterol aggregation, which make the plaque unstable through the formation of the necrotic lipid core. Furthermore, the release of metalloproteinase from macrophages could weaken the fibrous cap of the plaque and promote rupture. ${ }^{35}$ Therefore, the antiapoptosis effects of macrophages in early-stage atherosclerotic lesions increase the likelihood of plaque development. ${ }^{34}$ Our group previously confirmed that PpIX could enter athermanous plaques in the rabbit carotid artery. ${ }^{5}$ We found that PpIX mainly targeted and accumulated in metabolically active inflammatory macrophages in the athermanous plaques of $\mathrm{ApoE}^{-/-}$mice (data not shown). In the present study, we focused on the use of SDT to induce macrophage apoptosis to stabilize the plaques.

Our group has previously shown that the application of different ultrasound intensities combined with diverse sonosensitizers produces similar apoptotic effects on macrophages through distinct mechanisms. Following emodin-SDT, the cytoskeleton loses its original features. For example, actin filaments disperse, and cytoskeletal proteins aggregate. ${ }^{21}$ During curcumin-SDT, ALA-SDT, and PpIX-SDT, $\Delta \Psi \mathrm{m}$ decreases, and there is a considerable increase in ROS, leading to apoptotic cell morphologies. ${ }^{1,14,17}$ Furthermore, under hydroxylacetylated curcumin-SDT, increasing ROS levels cause macrophage apoptosis through the caspase pathway. ${ }^{18}$ In these studies, different sonosensitizers were shown to accumulate in macrophages and to cause apoptosis when combined with ultrasound in vitro. Because low-intensity ultrasound induces little damage to tissue compared with high-intensity ultrasound, we employed a lower intensity of $0.5 \mathrm{~W} / \mathrm{cm}^{2}$, rather than the intensity of $2.0 \mathrm{~W} / \mathrm{cm}^{2}$ used in previous in vitro ${ }^{1,21}$ studies.

The time point at which the highest accumulation of $\mathrm{HY}$ occurs in THP-1 macrophages may be the optimum time to induce a burst of apoptosis via HY-SDT. The in vitro data suggested that HY entered THP-1 macrophages in a timedependent manner (Figure 2B) and reached a relatively high level after 4 hours of incubation. Possible mechanisms for the entry of HY into macrophages include passive diffusion, hydrophobicity (which is the main driving force for attraction to cell membranes due to their lipid composition), ${ }^{38}$ and phagocytosis. ${ }^{39}$ The CCK-8 results showed that $0.25 \mu \mathrm{g} / \mathrm{mL}$ HY was the optimal sonosensitizer concentration because, at this concentration, ultrasound could effectively induce THP-1 macrophage death (Figure 2C). At this HY concentration, cell viability was dependent on the ultrasound exposure time; this result indicated that a specific safe drug concentration and a specific ultrasound exposure time were necessary to evaluate the effects of SDT. Therefore, $0.25 \mu \mathrm{g} / \mathrm{mL} \mathrm{HY}$ and 15 minutes of ultrasound exposure were considered to represent the optimal conditions in this experiment for inducing efficient macrophage death in vitro. The $0.25 \mu \mathrm{g} / \mathrm{mL} H Y$ concentration was lower than the concentrations of emodin $(15 \mu \mathrm{g} / \mathrm{mL}),{ }^{21}$ curcumin $(15 \mu \mathrm{g} / \mathrm{mL}),{ }^{1}$ and ALA $(131.1 \mu \mathrm{g} / \mathrm{mL})^{14,17,18}$ required to obtain a similar sonodynamic effect. The present findings clearly indicate that HY is a more efficient sonosensitizer than those employed previously. ${ }^{1,19,21}$

The effects of SDT depend not only on the sonosensitizer concentration and the ultrasound activating time but also on the localization within THP-1 macrophages. Most radical products have very short lifetimes and diffusion ranges; those derived from sonosensitizers determine the localization of the primary damage and apoptotic triggers during the SDT process. Additionally, Theodossiou et $\mathrm{al}^{40}$ confirmed that exogenously administered HY primarily localizes to mitochondria. In this study, we also found that HY mainly localized to the mitochondria and Golgi apparatus and, to a lesser extent, to the nuclei (Figures 5 and 6); nuclear localization increased with increasing HY concentrations (Figure 5A). ALA-SDT could generate ROS changing the VDAC1 channel properties in outer mitochondrial membrane, and then affected ER status to induce the macrophages apoptosis. ${ }^{41}$ Wang et $\mathrm{al}^{18}$ demonstrated that ALA-PpIX mainly accumulated in mitochondria and ER, and mitochondria played the key role in inducing the foam cell apoptosis. HY might partly induce macrophage apoptosis through ER, except mitochondria. The large accumulation of HY in Golgi apparatus might be related to the synthesis of apoptosis-related proteins, while the detailed mechanism needs to be further explored. Recently, Ouimet et $\mathrm{al}^{42}$ proved that lipid droplets in macrophage foam cells could be delivered to lysosomes by autophagy in which lysosomal acid lipase hydrolyze them to free cholesterol. HY partly accumulated in lysosome might indicate that HY-SDT could stimulate the autophagy generation; the condition of HY-SDT in this study was not appropriate for autophagy but was for apoptosis. 
As was previously mentioned, the CCK- 8 assay suggested that HY-SDT decreased cell viability but did not indicate the mode of cell death. Clinically, apoptosis is considered to be more desirable than necrosis because it triggers less inflammation, fewer side effects, and fewer other immune reactions. ${ }^{43,44}$ In this study, the mode of cell death was determined through a Hoechst-PI assay, transmission electron microscopy, and Western blot analyses of apoptosis-related proteins. The results indicated that HY-SDT could induce both apoptosis and necrosis, and apoptosis was the predominant mode of cell death (Figure 3A). Ultrastructural changes (Figure 3B) in macrophages showed that the cells affected by SDT displayed apoptotic features, and the mitochondria were the most activated organelles. This result was consistent with previous SDT results showing that apoptosis is induced via the mitochondrial pathway. ${ }^{45,46}$ The expression of BAX was upregulated, and the expression of Bcl-2 was downregulated (Figure 3C). Meanwhile, the expression levels of the apoptosis-related proteins cleaved caspase-9, cleaved caspase-3, and cleaved PARP were increased upon SDT (Figure 3D). Moreover, the broad-spectrum caspase inhibitor $\mathrm{z}-\mathrm{VAD}$ prevented the death of THP-1 macrophages caused by SDT (Figure 3E). Based on these results, it can be concluded that HY-SDT induced THP-1 macrophage apoptosis through initial injury to the mitochondria; this pathway clearly represents a different mechanism from those involved in other types of sonosensitizer-mediated SDT. ${ }^{1,21}$

A critical step in apoptosis is mitochondrial membrane permeabilization, indicating the loss of mitochondrial membrane integrity. ${ }^{19}$ This step results in the release of proteins that are critical for downstream events and constitutes a no return point in cell death. We confirmed that HY primarily accumulated in the mitochondria of THP-1 macrophages. Therefore, we detected mitochondrial dysfunction by monitoring $\Delta \Psi \mathrm{m}$ and observed a significant decrease in $\Delta \Psi \mathrm{m}$ after SDT (Figure $7 \mathrm{~F}$ ). This result was in agreement with our previous studies, indicating that the mitochondria might be the most effective damage sites to target in SDT..$^{14,17}$ Our group previously confirmed that ultrasound-activated sonosensitizers at the binding site can significantly enhance intracellular ROS levels, which are responsible for the primary cytotoxic mechanism involved in SDT, rather than mechanical shear stresses or thermal effects. ${ }^{1,14,17-19,21}$ In the present study, we found that HY-SDT could increase the levels of ROS (Figure 4C). Both the ROS scavenger $\mathrm{NAC}$ and the singlet oxygen scavenger $\mathrm{NaN}_{3}$ significantly attenuated the generation of ROS. Furthermore, we chose other scavengers, including mannitol (hydroxyl radicals),
SOD (superoxide anion radicals), and CAT (hydrogen peroxide), to determine the type of ROS primarily involved in SDT. The pretreatment of cultures with NAC and $\mathrm{NaN}_{3}$ not only significantly reduced ROS generation but also rescued the SDT-induced macrophage cytotoxicity (Figure 4A-C). Moreover, to confirm the relationship between ROS and a decreased $\Delta \Psi \mathrm{m}, \Delta \Psi \mathrm{m}$ was measured in cells pretreated with NAC and $\mathrm{NaN}_{3}$ prior to HY-SDT. NAC and $\mathrm{NaN}_{3}$ were observed to significantly reverse the decrease in $\triangle \Psi \mathrm{m}$ caused by HY-SDT. These results implied that singlet oxygen might be the primary ROS acting as an upstream regulator to cause mitochondrial damage, leading to cell apoptosis post-SDT. This predicted mechanism is consistent with those indicated in previous studies. ${ }^{17,46}$

The $\Delta \Psi \mathrm{m}$ of the CsA group was increased relative to that of the SDT group, which indicated that HY-SDT-mediated apoptosis might be due to a reduction of $\Delta \Psi \mathrm{m}$ via targeting of mPTP. This hypothesis was in agreement with previous studies. ${ }^{17,47} \mathrm{Next}$, we found that the mPTP opened after SDT; however, this effect was partially blocked by pretreatment with CsA, rather than BA and DIDS (Figure 7C). Therefore, we speculated that cyclophilin D, as a member of the MPTP, might be the most crucial factor responsible for MPTP opening during HY-SDT.

Furthermore, we determined the contents of MDA, which is the end product of lipid peroxidation, in the mitochondrial membrane after different treatments. The SDT group was observed to present a high MDA content (Figure 7E), which might be due to the much higher level of ROS in the SDT group, which further attack polyunsaturated fatty acids in mitochondrial membrane lipids. The occurrence of lipid peroxidation due to oxidative stress might alter the structure of the mitochondrial membrane, affect its physiological function, open the mPTP, and, finally, lead to apoptosis. ${ }^{48,49}$

The activation of apoptosis-related proteins is the most conclusive event during apoptosis. Cytochrome $\mathrm{C}$ is released from the intermembrane space of mitochondria into the cytosol and activates the caspase- 9 proenzyme, which, in turn, activates caspase-3, which cleaves PARP to initiate a caspase cascade leading to apoptosis. ${ }^{29,50}$ Our results were in agreement with those of previous studies. We also observed that the translocation of BAX and cytochrome $\mathrm{C}$ might be because $\mathrm{BAX}$ proteins anchor to the mitochondria, whereas cytochrome $\mathrm{C}$ is released following SDT. ${ }^{47}$ According to these results, HY-SDT induces THP-1 macrophage apoptosis via the mitochondria-caspase pathway, activated by BAX and cytochrome $\mathrm{C}$ translocation, leading to an increase in the ratio between BAX and Bcl-2. 
This study demonstrated the potential use of HY-SDT for treating AS. However, given the complicated role of macrophages in AS, additional studies addressing HY-SDT in animal AS models should be performed.

\section{Conclusion}

The present study offers strong evidence suggesting that HY-SDT induces the apoptosis of THP-1 macrophages in vitro, partly by targeting the mitochondria, through singlet oxygen (the main ROS) generation, thereby oxidizing mitochondrial membrane lipids. The subsequent translocation of BAX and cytochrome $\mathrm{C}$ increases the ratio of BAX to $\mathrm{Bcl}-2$, causing $\mathrm{mPTP}$ opening to trigger the mitochondriacaspase pathway.

\section{Acknowledgments}

This study was supported by the National Natural Science Foundation of China $(81000688,81271734)$, the Heilongjiang Provincial Youth Science Foundation (QC2011C002), the Foundation of Science and Technology Innovation Talent of Harbin Science and Technology Bureau (2011RFQXS072), the Wu Lian de Youth Science Foundation of Harbin Medical University (WLD-QN1104), the Postdoctoral Science-Research Developmental Foundation of Heilongjiang Province (LBHQ12049), the China Postdoctoral Science Foundation funded project (20090460911), the Ministry of Education, and the Research Foundation of the Health Department of Heilongjiang Province (2009-217).

\section{Disclosure}

There are no conflicts of interest among the authors of this work.

\section{References}

1. Wang FP, Gao QP, Guo SY, et al. The sonodynamic effect of curcumin on THP-1 cell-derived macrophages. Biomed Res Int. 2013;2013: 737264.

2. Moore KJ, Sheedy FJ, Fisher EA. Macrophages in atherosclerosis: a dynamic balance. Nat Rev Immunol. 2013;13(10):709-721.

3. Tang G, Hyman S, Schneider JH Jr, et al. Application of photodynamic therapy to the treatment of atherosclerotic plaques. Neurosurgery. 1993;32(3):438-443.

4. Waksman R, McEwan PE, Moore TI, et al. PhotoPoint photodynamic therapy promotes stabilization of atherosclerotic plaques and inhibits plaque progression. J Am Coll Cardiol. 2008;52(12):1024-1032.

5. Peng C, Li Y, Liang H, et al. Detection and photodynamic therapy of inflamed atherosclerotic plaques in the carotid artery of rabbits. J Photochphotobio B. 2011;102(1):26-31.

6. Qiang YG, Yow C, Huang Z. Combination of photodynamic therapy and immunomodulation: current status and future trends. Med Res Rev. 2008;28(4):632-644.

7. Tachibana K, Feril LB Jr, Ikeda-Dantsuji Y. Sonodynamic therapy. Ultrasonics. 2008;48(4):253-259.
8. Umemura S, Yumita N, Nishigaki R. Enhancement of ultrasonically induced cell damage by a gallium-porphyrin complex, ATX-70. Jpn J Cancer Res. 1993;84(5):582-588.

9. Kuroki M, Hachimine K, Abe H, et al. Sonodynamic therapy of cancer using novel sonosensitizers. Anticancer Res. 2007;27:3673-3677.

10. Ninomiya K, Noda K, Ogino C, et al. Enhanced OH radical generation by dual-frequency ultrasound with $\mathrm{TiO}_{2}$ nanoparticles: Its application to targeted sonodynamic therapy. Ultrason Sonochem. 2014;21(1): 289-294.

11. Agostinis P, Berg K, Cengel KA, et al. Photodynamic therapy of cancer: an update. CA Cancer J Clin. 2011;61(4):250-281.

12. Rosenthal I, Sostaric JZ, Riesz P. Sonodynamic therapy: a review of the synergistic effects of drugs and ultrasound. Ultrason Sonochem. 2004;11(6):349-363.

13. Bai WK, Shen E, Hu B. Induction of the apoptosis of cancer cell by sonodynamic therapy: a review. Chiense J Cancer Res. 2012;24(4): $368-373$.

14. Guo S, Sun X, Cheng J, et al. Apoptosis of THP-1 macrophages induced by protoporphyrin IX-mediated sonodynamic therapy. Int J Nanomed. 2013; 8:2239-2246.

15. He Y, Xia X, Xu C, et al. 5-Aminolaevulinic acid enhances ultrasoundinduced mitochondrial damage in K562 cells. Ultrasonics. 2010;50(8): $777-781$.

16. Wang X, Leung AW, Jiang Y, et al. Hypocrellin B-mediated sonodynamic action induces apoptosis of hepatocellular carcinoma cells. Ultrasonics. 2012;52(4):543-546.

17. Cheng J, Sun X, Guo S, et al. Effects of 5-aminolevulinic acid-mediated sonodynamic therapy on macrophages. Int J Nanomed. 2013;8: 669.

18. Wang H, Yang Y, Chen H, et al. The predominant pathway of apoptosis in THP-1 macrophage-derived foam cells induced by 5 -aminolevulinic acid-mediated sonodynamic therapy is the mitochondria-caspase pathway despite the participation of endoplasmic reticulum stress. Cell Physiol Biochem. 2014;33(6):1789-1801.

19. Zheng L, Sun X, Zhu X, et al. Apoptosis of THP-1 derived macrophages induced by sonodynamic therapy using a new sonosensitizer hydroxyl acetylated curcumin. PloS One. 2014;9(3):e93133.

20. Wang X, Guo Y, Yang S, et al. Cellular and molecular mechanisms of photodynamic hypericin therapy for nasopharyngeal carcinoma cells. J Pharmacol Exp Ther. 2010;334(3):847-853.

21. Gao Q, Wang F, Guo S, et al. Sonodynamic effect of an anti-inflammatory agent-emodin on macrophages. Ultrasound Med Biol. 2011;37(9): 1478-1485.

22. Sarris J, Panossian A, Schweitzer I, et al. Herbal medicine for depression, anxiety and insomnia: a review of psychopharmacology and clinical evidence. Eur Neuropsychopharm. 2011;21(12):841-860.

23. Ehrenshaft M, Roberts JE, Mason RP. Hypericin-mediated photooxidative damage of $\alpha$-crystallin in human lens epithelial cells. Free Radical Bio Med. 2013;60:347-354.

24. Božin B, Kladar N, Grujić N, et al. Impact of origin and biological source on chemical composition, anticholinesterase and antioxidant properties of some St. John's wort species (Hypericum spp., Hypericaceae) from the Central Balkans. Molecules. 2013;18(10):11733-11750.

25. Theodossiou TA, Hothersall JS, De Witte PA, et al. The multifaceted photocytotoxic profile of hypericin. Mol Pharm. 2009;6(6): 1775-1789.

26. Sosa S, Pace R, Bornanciny A, et al. Topical anti-inflammatory activity of extracts and compounds from Hypericum perforatum L. J Pharm Pharmacol. 2007;59(5):703-709.

27. Vacek J, Klejdus B, Kuban V. [Hypericin and hyperforin: bioactive components of St. John's wort (Hypericum perforatum). Their isolation, analysis and study of physiological effect]. Ceska Slov Farm. 2007;56(2):62-66. Czech.

28. Huntosova V, Nadova Z, Dzurova L, et al. Cell death response of U87 glioma cells on hypericin photoactivation is mediated by dynamics of hypericin subcellular distribution and its aggregation in cellular organelles. Photoch Photobio Sci. 2012;11(9):1428-1436. 
29. Chou LC, Yang JS, Huang LJ, et al. The synthesized 2-(2fluorophenyl)-6, 7-methylenedioxyquinolin-4-one (CHM-1) promoted G2/M arrest through inhibition of CDK1 and induced apoptosis through the mitochondrial-dependent pathway in CT-26 murine colorectal adenocarcinoma cells. J Gastroenterol. 2009;44(10):1055-1063.

30. Song W, Cui H, Zhang R, et al. Apoptosis of SAS cells induced by sonodynamic therapy using 5-aminolevulinic acid sonosensitizer. Anticancer Res. 2011;31(1):39-45.

31. Tsuru H, Shibaguchi H, Kuroki M, et al. Tumor growth inhibition by sonodynamic therapy using a novel sonosensitizer. Free Radical Bio Med. 2012;53(3):464-472.

32. Wang S, Wang Q, Wang Y, et al. Novel anthraquinone derivatives: synthesis via click chemistry approach and their induction of apoptosis in BGC gastric cancer cells via reactive oxygen species (ROS)dependent mitochondrial pathway. Bioorg Med Chem Lett. 2008;18(24): 6505-6508.

33. Rocha VZ, Libby P. Obesity, inflammation, and atherosclerosis. Nat ReV Cardiol. 2009;6(6):399-409.

34. Gautier EL, Huby T, Witztum JL, et al. Macrophage apoptosis exerts divergent effects on atherogenesis as a function of lesion stage. Circulation. 2009;119(13):1795-1804.

35. Estronca LMBB, Silva JCP, Sampaio JL, et al. Molecular etiology of atherogenesis-in vitro induction of lipidosis in macrophages with a new LDL model. PloS One. 2012;7(4):e34822.

36. Robbins CS, Hilgendorf I, Weber GF, et al. Local proliferation dominates lesional macrophage accumulation in atherosclerosis. Nat Med. 2013;19(9):1166-1172.

37. Barati AH, Mokhtari-Dizaji M, Mozdarani H, et al. Treatment of murine tumors using dual-frequency ultrasound in an experimental in vivo model. Ultrasound Med Biol. 2009;35(5):756-763.

38. Ho YF, Wu MH, Cheng BH, et al. Lipid-mediated preferential localization of hypericin in lipid membranes. Biochim Biophys Acta. 2009; 1788(6):1287-1295.

39. Moore KJ, Tabas I. Macrophages in the pathogenesis of atherosclerosis. Cell. 2011;145(3):341-355.
40. Theodossiou TA, Noronha-Dutra A, Hothersall JS. Mitochondria are a primary target of hypericin phototoxicity: synergy of intracellular calcium mobilisation in cell killing. Int J Biochem Cell B. 2006;38(11): 1946-1956.

41. Chen H, Yang Y, Guo S, et al. Inhibition of VDAC1 prevents $\mathrm{Ca}^{2+}$ mediated oxidative stress and apoptosis induced by 5 -aminolevulinic acid mediated sonodynamic therapy in THP-1 macrophages. Apoptosis. 2014;19(12):1712-1726.

42. Ouimet M, Franklin V, Mak E, Liao X, Tabas I, Marcel YL. Autophagy regulates cholesterol efflux from macrophage foam cells via lysosomal acid lipase. Cell Metab. 2011;13:655-667.

43. Martinet W, Verheye S, De Meyer GRY. Selective depletion of macrophages in atherosclerotic plaques via macrophage-specific initiation of cell death. Trends Cardiovas Med. 2007;17(2):69-75.

44. Tabas I. Macrophage death and defective inflammation resolution in atherosclerosis. Nat Rev Immunol. 2009;10(1):36-46.

45. Tang W, Liu Q, Zhang J, et al. In vitro activation of mitochondriacaspase signaling pathway in sonodynamic therapy-induced apoptosis in sarcoma 180 cells. Ultrasonics. 2010;50(6):567-576.

46. Su X, Li Y, Wang P, et al. Protoporphyrin IX-mediated sonodynamic action induces apoptosis of K562 cells. Ultrasonics. 2014;54(1): 275-284.

47. Tiwari BS, Belenghi B, Levine A. Oxidative stress increased respiration and generation of reactive oxygen species, resulting in ATP depletion, opening of mitochondrial permeability transition, and programmed cell death. Plant Physiol. 2002;128(4):1271-1281.

48. Jin H, Zhong X, Wang Z, et al. Sonodynamic effects of hematoporphyrin monomethyl ether on CNE-2 cells detected by atomic force microscopy. J Cell Biochem. 2011;112(1):169-178.

49. Li JH, Yue W, Huang Z, et al. Calcium overload induces C6 rat glioma cell apoptosis in sonodynamic therapy. Int J Radiat Biol. 2011;87(10): 1061-1066.

50. Sheridan C, Martin SJ. Mitochondrial fission/fusion dynamics and apoptosis. Mitochondrion. 2010;10(6):640-648.
International Journal of Nanomedicine

\section{Publish your work in this journal}

The International Journal of Nanomedicine is an international, peerreviewed journal focusing on the application of nanotechnology in diagnostics, therapeutics, and drug delivery systems throughout the biomedical field. This journal is indexed on PubMed Central, MedLine, CAS, SciSearch ${ }^{\circledR}$, Current Contents ${ }^{\circledR} /$ Clinical Medicine,

\section{Dovepress}

Journal Citation Reports/Science Edition, EMBase, Scopus and the Elsevier Bibliographic databases. The manuscript management system is completely online and includes a very quick and fair peer-review system, which is all easy to use. Visit http://www.dovepress.com/ testimonials.php to read real quotes from published authors. 